\title{
Oxidative stress, mitochondrial damage, and cores in muscle from calsequestrin-1 knockout mice
}

\author{
Cecilia Paolini ${ }^{1}$, Marco Quarta ${ }^{2,4}$, Lan Wei-LaPierre ${ }^{3}$, Antonio Michelucci ${ }^{1}$, Alessandra Nori ${ }^{2}$, Carlo Reggiani ${ }^{2}$,
} Robert T Dirksen ${ }^{3}$ and Feliciano Protasi ${ }^{*}$

\begin{abstract}
Background: Mutations in the gene encoding ryanodine receptor type-1 (RYR1), the calcium ion $\left(\mathrm{Ca}^{2+}\right)$ release channel in the sarcoplasmic reticulum (SR) of skeletal muscle, are linked to central core disease (CCD) and malignant hyperthermia $(\mathrm{MH})$ susceptibility. We recently reported that mice lacking the skeletal isoform of calsequestrin (CASQ1-null), the primary $\mathrm{Ca}^{2+}$ buffer in the SR of skeletal muscle and a modulator of RYR1 activity, exhibit lethal heat- and anesthetic-induced hypermetabolic episodes that resemble $\mathrm{MH}$ events in humans.
\end{abstract}

Methods: We compared ultrastructure, oxidative status, and contractile function in skeletal fibers of extensor digitorum longus (EDL) muscles in wild type (WT) and CASQ1-null mice at different ages (from 4 to 27 months) using structural, biochemical, and functional assays.

Results: About 25\% of fibers in EDL muscles from CASQ1-null mice of 14 to 27 months of age exhibited large areas of structural disarray (named core-like regions), which were rarely observed in muscle from age-matched WT mice. To determine early events that may lead to the formation of cores, we analyzed EDL muscles from adult mice: at 4 to 6 months of age, CASQ1-null mice (compared to WT) displayed significantly reduced grip strength ( $40 \pm 1$ vs. $86 \pm 1 \mathrm{mN} / \mathrm{gr}$ ) and exhibited an increase in the percentage of damaged mitochondria (15.1\% vs. 2.6\%) and a decrease in average cross-sectional fiber area (approximately 37\%) in EDL fibers. Finally, oxidative stress was also significantly increased (25\% reduction in ratio between reduced and oxidized glutathione, or GSH/GSSG, and 35\% increase in production of mitochondrial superoxide flashes). Providing ad libitum access to $\mathrm{N}$-acetylcysteine in the drinking water for 2 months normalized GSH/GSSG ratio, reduced mitochondrial damage (down to 8.9\%), and improved grip strength (from $46 \pm 3$ to $59 \pm 2 \mathrm{mN} / \mathrm{gr}$ ) in CASQ1-null mice.

Conclusions: Our findings: 1) demonstrate that ablation of CASQ1 leads to enhanced oxidative stress, mitochondrial damage, and the formation of structural cores in skeletal muscle; 2) provide new insights in the pathogenic mechanisms that lead to damage/disappearance of mitochondria in cores; and 3) suggest that antioxidants may provide some therapeutic benefit in reducing mitochondrial damage, limiting the development of cores, and improving muscle function.

Keywords: Excitation-contraction (EC) coupling, Mitochondria, Ryanodine receptor (RYR)

\section{Background}

In skeletal muscle, excitation-contraction (EC) coupling is a process by which depolarization of the transverse (T)-tubule membrane is mechanically linked to the release of calcium ions $\left(\mathrm{Ca}^{2+}\right)$ from the sarcoplasmic reticulum (SR) and, hence, activation of muscle contraction [1]. EC

\footnotetext{
* Correspondence: fprotasi@unich.it

${ }^{1}$ CeSI - Center for Research on Ageing \& DNICS - Department of Neuroscience, Imaging and Clinical Sciences, University G. d'Annunzio, Via L. Polacchi, 11, I-66013 Chieti, Italy

Full list of author information is available at the end of the article
}

coupling in skeletal muscle involves direct, bi-directional communication between T-tubule voltage sensors (dihydropyridine receptors, $\left.\alpha_{1 \mathrm{~s}} \mathrm{DHPRs}\right)$ and $\mathrm{SR} \mathrm{Ca}^{2+}$ release channels (ryanodine receptor type-1, RYR1) [2,3]. Several other junctional proteins, including calsequestrin-1, triadin, junctin, JP-45, stac3, and FK-506 binding protein, interact with RYR1 and $\alpha_{1} \mathrm{sDHPR}$ to form a macromolecular machinery that controls $\mathrm{Ca}^{2+}$ release [4]. Several pathological conditions that adversely affect striated muscle function are associated with mutations in EC

\section{Biomed Central}


coupling proteins that result in alterations in the proper control of SR $\mathrm{Ca}^{2+}$ release [5].

Mutations in the RYR1 gene account for the majority of cases of malignant hyperthermia susceptibility (MHS) and central core disease (CCD) in humans [6-8]. CCD is one of the most common congenital myopathies: $\mathrm{CCD}$ patients exhibit fetal hypotonia, proximal muscle weakness, and a significant delay in motor development [9,10]. Diagnosis of CCD is confirmed by histological examination of muscle biopsies showing amorphous central areas (or cores, typically found in type I fibers) lacking glycolytic/oxidative enzymes and mitochondria [11]. Disorganization of the contractile elements and sarco-tubular systems is typically observed within the core regions [12]. MHS, on the other hand, is a pharmacogenetic disorder characterized by life-threatening episodes triggered by volatile anesthetics (for example, halothane, isoflurane, etc.) and depolarizing muscle relaxants (for example, succinylcholine) [13-15] that occurs in the absence of an overt myopathy. CCD and MHS are often related: some patients with CCD test positive for MHS $[16,10]$ and, thus, are considered at risk for malignant hyperthermia $(\mathrm{MH})$ [17].

Calsequestrin (CASQ) is a highly acidic protein that binds $\mathrm{Ca}^{2+}$ that is concentrated at the junctional face of the terminal cisternae, near the sites of RYR $1 \mathrm{Ca}^{2+}$ release [18-20]. There are two isoforms of mammalian CASQ that are products of different genes: the skeletal (CASQ1) and cardiac (CASQ2) isoforms, which are expressed at different levels during development of fast- and slow-twitch skeletal muscle fibers [21,22]. In addition to being important for SR $\mathrm{Ca}^{2+}$ binding/storage, CASQ1 also modulates the RYR1 $\mathrm{Ca}^{2+}$ release channel activity $[23,24]$.

Our structural and functional studies in CASQ1knockout (or null) mice revealed that while CASQ1 ablation is not lethal [25], skeletal muscle from CASQ1null mice exhibits significant structural remodeling of $\mathrm{Ca}^{2+}$ release units (CRUs) and impaired $\mathrm{Ca}^{2+}$ handling [25-27]. Abnormal CRUs (that is, forming multiple elements with reduced junctional SR lumen) exhibit rapid and severe $\mathrm{SR} \mathrm{Ca}^{2+}$ depletion in response to repetitive stimulation [28]. As a result, EC coupling is unable to support prolonged $\mathrm{Ca}^{2+}$ transients required to sustain force production during tetanic stimulation $[26,28]$. Importantly, CASQ1-null mice also exhibit life-threatening, hypermetabolic episodes characterized by sustained muscle contractures, rhabdomyolysis, and uncontrolled elevations in core body temperature when exposed to either halothane or environmental heat stress [29-31], which closely resemble human MHS [14,15]. However, mutations in the CASQ1 gene have not been identified in $\mathrm{MH}$ patients. In fact, Kraeva et al. [32] failed to identify any mutations within the CASQ1 coding region in 75 unrelated MHS patients diagnosed by caffeine-halothane contracture test, thus concluding that CASQ1 is an unlikely genetic locus for MHS within the North American population [33]. A similar conclusion was reached in another study of European MHS patients (Vincenzo Sorrentino, University of Siena, personal communication). However, a missense mutation in the CASQ1 gene (N244G, the first CASQ1 disease mutation identified in humans) was recently reported in a group of patients with a myopathy characterized by weakness, fatigue, and the presence of large vacuoles containing characteristic inclusions resulting from the aggregation of SR proteins [34]. The MH status of individuals that possess the N244G mutation in CASQ1 is currently unknown.

Since CASQ1 exhibits an MHS-like phenotype and MHS occurs in some CCD patients, we hypothesized that CASQ1-null mice may develop a myopathy resembling CCD. To test this hypothesis, we compared skeletal muscle ultrastructure, oxidative status, and contractile function in wild type (WT) and CASQ1-null mice at different ages ( 4 to $6,14,20,24$, and 27 months). Our results indicate that CASQ1-null mice exhibit impaired muscle function (that is, reduced force output), mitochondrial damage, and structural core-like regions similar to that described previously in RYR1 mouse models of $\mathrm{MH} / \mathrm{CCD}[35,36]$. We found that increased oxidative stress appears to be a key trigger for this myopathic phenotype in CASQ1-null mice as treatment with the antioxidant $\mathrm{N}$-acetylcysteine (NAC) provides significant protection from the development of structural and functional deficits.

\section{Methods}

All functional, structural, and molecular analyses were carried out in either a) extensor digitorum longus (EDL; predominantly fast-twitch fibers), the muscle group which is more affected by CASQ1 ablation $[25,26]$, or b) flexor digitorum brevis (FDB; approximately $80 \%$ of fasttwitch fibers; [37]) for single cell experiments.

\section{Ethics statement}

All experiments were conducted according to the Directive of the European Union 2010/63/UE and the National Institutes of Health Guide for the Care and Use of Laboratory Animals. All animal protocols were approved by the Committee on the Ethics of Animal Experiments of the University of Chieti (CEISA; Permit Number: 40) and the University Committee on Animal Resources at the University of Rochester Medical Center. All surgeries were made to minimize animal suffering: animals were anesthetized and then sacrificed by cervical dislocation.

\section{CASQ1-null mice}

CASQ1-null mice [25] were housed in micro-isolator cages, at $20^{\circ} \mathrm{C}$ in a 12 -h light/dark cycle, with free access 
to water and food. As life expectancy of CASQ1-null male mice is significantly reduced (see Additional file 1 : Figure S1; see also refs. [29,31]), all experiments conducted in this study were performed using female WT and CASQ1-null animals (due to shortage of aged male CASQ1-null mice). For in vivo antioxidant treatment, mice were provided ad libitum access to drinking water containing NAC $(1 \% w / v)$.

\section{Grip strength test}

Grip strength of WT and CASQ1-null mice from three different age groups (4 to 6, 14, and 22 to 24 months of age) was measured as described by Connolly et al. [38]. Briefly, mice were held by the tail and lowered to a metal grating connected to the shaft of a Shimpo Fgv 0.5X force transducer (Metrotec Group, San Sebastián, Spain). Once the mouse had firmly grabbed the grating, a gentle pull was exerted on the tail. Measurements of peak force generated by each mouse using fore and hind limbs were repeated three times with appropriate intervals (at least 30 s) to avoid fatigue. Average peak force values were normalized to total body mass measured immediately before each experiment.

\section{Force and contraction kinetics of isolated EDL muscles}

EDL muscles were dissected from the hind limb of WT and CASQ1-null mice of three different age groups (4 to 6,14 , and 22 to 24 months old). Force and contraction kinetics were measured as described by Paolini et al. [25]. Excised muscles were continuously perfused with oxygenated Krebs solution $(\mathrm{NaCl} 118 \mathrm{mM}, \mathrm{KCl} 4.7 \mathrm{mM}$, $\mathrm{MgSO}_{4} 1.2 \mathrm{mM}, \mathrm{KH}_{2} \mathrm{PO}_{4} 1.1 \mathrm{mM}$, glucose $11.1 \mathrm{mM}$, $\mathrm{CaCl}_{2} 2.5 \mathrm{mM}, \mathrm{NaHCO}_{3} 25 \mathrm{mM}, \mathrm{pH}$ 7.4) and mounted between a force transducer (AME-801 SensorOne, Sausalito, CA, USA) and micromanipulator-controlled shaft in a small chamber. The temperature of the chamber was maintained at $25^{\circ} \mathrm{C}$. The stimulation conditions were optimized, and muscle length was increased until force development during tetanus was maximal. Single twitches and fused tetani $(120 \mathrm{~Hz}, 0.5-$ $\mathrm{s}$ duration) under isometric conditions were recorded with rest intervals suitable to avoid fatigue. Specific force $\left(\mathrm{mN} / \mathrm{mm}^{2}\right)$ and time to peak contraction (s) were measured.

\section{Preparation of samples for histology and EM}

EDL muscles were carefully dissected from WT and CASQ1-null mice at different ages (4 to 6, 14, 20, 24, and 27 months). Muscles were fixed at room temperature (RT) in $3.5 \%$ glutaraldehyde in $0.1 \mathrm{M}$ sodium cacodylate buffer, $\mathrm{pH} 7.2$, for $2 \mathrm{~h}$ and kept in fixative until further use. Small bundles of fibers were then postfixed and embedded as described by Paolini et al. [25]. For histological analyses, longitudinal and cross-oriented semithin sections $(250 \mathrm{~nm})$ were cut with a Leica Ultracut $\mathrm{R}$ microtome (Leica Microsystem, Vienna, Austria) using a Diatome diamond knife (Diatome Ltd. CH2501 Biel, Switzerland). After staining with toluidine blue dye, the sections were viewed on a Leica DMLB fluorescence microscope (Leica Microsystem, Vienna, Austria). For electron microscopy (EM), ultrathin sections were cut (approximately $50 \mathrm{~nm}$ ), stained in $4 \%$ uranyl acetate and lead citrate, and examined with a Morgagni Series 268D electron microscope (FEI Company, Brno, Czech Republic) equipped with a Megaview III digital camera.

\section{Quantitative analyses of histological and EM specimens}

1) Measurements of fiber cross-sectional area (CSA) were performed on cross-oriented semithin sections of EDL muscles from WT and CASQ1-null mice of 4 to 6 , 14 , and 24 months of age (by histological analysis). Images were selected and CSA calculated using the AnalySIS software (Olympus Soft Imaging Solutions GmbH, Munster, Germany). 2) Mitochondrial volume was determined in adult (4 to 6 months) and aged (27 months) WT and CASQ1-null mice before and after NAC treatment. Mitochondrial volume was calculated using the wellestablished stereology point-counting technique $[39,40]$ in EM micrographs taken at $14,000 \times$ of magnification after superimposing an orthogonal array of dots at a spacing of $0.20 \mu \mathrm{m}$ to the electron micrographs. The ratio between the number of dots falling within the mitochondrial profile and the total number of dots covering the whole image was used to calculate the relative volume of the fiber occupied by mitochondria. 3) The number of altered mitochondria was determined in EDL muscles from 4to 6-month-old WT and CASQ1-null mice (in the absence or presence of NAC treatment) using the same set of EM micrographs as described for the mitochondrial volume measurements. Mitochondria with any one (or more) of the following ultrastructural alterations were classified as damaged: a) swollen mitochondria, b) mitochondria with clear disruption of the external membrane and/or internal cristae, c) mitochondria containing vacuoles, and d) mitochondria containing myelin figures. The quantification was reported as the percentage of all mitochondria evaluated in the fiber. 4) Determination of the number of damaged fibers in adult (4-monthold) and aged (four time points between 14 and 27 months of age) mice was performed on histological sections stained with toluidine blue. Quantitative analysis was performed at multiple time points for each genotype. Individual fibers were visually scored for the presence of unstructured cores and contracture cores, as described in Boncompagni et al. 2009 [35]. The number of fibers with alterations was presented as a percentage of all fibers evaluated. 


\section{Immunohistochemistry}

Dissected EDL muscles from 27-month-old WT and CASQ1-null mice were fixed in 2\% paraformaldehyde in phosphate-buffered saline (PBS) for $20 \mathrm{~min}$ at RT. Small bundles of fibers were blocked for $1 \mathrm{~h}$ in PBS containing $1 \%$ bovine serum albumin (BSA), $10 \%$ goat serum, and $0.5 \%$ TRITON X-100 and then incubated overnight at $4{ }^{\circ} \mathrm{C}$ with primary antibodies. After washing in PBS/BSA, fibers were incubated for $1 \mathrm{~h}$ at RT with secondary antibodies, washed again, and finally mounted with an anti-bleach medium. Code, specificity, working dilution, and the sources of primary and secondary antibodies used were as follows: mouse anti-RYR 34C, 1:30 [41] (Developmental Studies Hybridoma Bank, The University of Iowa), and Cy3 goat anti-mouse, 1:200 (Jackson ImmunoResearch Laboratories, West Grove, PA, USA). The specimens were viewed using a laser-scanning confocal microscope interfaced with an inverted Zeiss Axiovert microscope (LSM510 META, Zeiss, Germany).

\section{Gene profile analyses}

Microarray analyses were performed by CRIBI - Biotechnology Center and Biology Department Padua University using spotted oligonucleotide Micro-CRIBI Mouse Oligo 13.4k Array V_0 (Operon Version 1.1), GEO accession platform number GPL6747. This microarray set consists of 13.440 70-mer oligonucleotides designed on Mouse Unigene clusters, mainly in the $3^{\prime}$ end terminal region. Briefly, each oligonucleotide is spotted by a robotic station Biorobotics Microgrid II, in one replicate on a MICROMAX glass slide SuperChip I (Cat No MPS696) provided by PerkinElmer Life Sciences Inc. The deposition is qualitatively assessed by a scan of the spotted slide at $10-\mu \mathrm{m}$ resolution, using a ScanArray LITE (PerkinElmer, Waltham, MA, USA). Code GSE25984 at: http://www.ncbi.nlm.nih. gov/geo/query/acc.cgi?token $=$ xdojfeeesguuwji\&acc $=$ GSE 25984.

For all genes, $P=0.05,-0,65>\operatorname{LogR}>0,65$.

\section{Western blot analysis}

Preparation of total homogenates from EDL muscles of 4-month-old female WT and CASQ1-null mice were performed as previously described [25]. Identification of peroxisome proliferator-activated receptor gamma coactivator 1-alpha (PGC-1 $\alpha$ ) was obtained in three different EDL muscles from three different animals using a polyclonal antibody (H-300 Santa Cruz Biotechnology, Dallas, TX, USA) diluted at 1:200. Immuno-detection was performed using an anti-rabbit alkaline phosphataseconjugated antibody at a 1:10.000 dilution. For densitometric analysis, scans of nitrocellulose membranes were quantified using Scion Image J Software (National Institutes of Health, Bethesda, MD, USA). Normalization to actin was performed using Ponceau $S$ red staining to assess total protein loading (100 $\mu \mathrm{g}$ per lane). Mean optical density (O.D.) values of PGC- $1 \alpha$ bands were normalized to that observed for WT muscles, set as 100 O.D., and data were presented as density ratio. As the PGC- $1 \alpha$ antibody detected a doublet in all samples, the two bands were compared with that of a positive control (A-673 nuclear extract from rhabdomyosarcoma cells, Santa Cruz Biotechnology, Dallas, TX, USA) to determine the band corresponding to PGC-1 $\alpha$ : PGC- $1 \alpha$ was identified as the lower band in the doublet observed upon Western blot analysis (see Additional file 2: Figure S2).

\section{In vivo electroporation of mt-cpYFP cDNA into hind limb footpads of anesthetized mice}

WT and CASQ1-null mice (4 to 5 months old) were anesthetized by intraperitoneal injection of $100 \mathrm{mg} / \mathrm{kg}$ ketamine, $10 \mathrm{mg} / \mathrm{kg}$ xylazine, and $3 \mathrm{mg} / \mathrm{kg}$ acepromazine. Hind limb footpads of anesthetized mice were then injected subcutaneously with bovine hyaluronidase $(7 \mu \mathrm{l} /$ foot, $2 \mu \mathrm{g} / \mu \mathrm{l})$. One hour later, hind limb footpads were subcutaneously injected with $30 \mu \mathrm{g}$ of mitochondriatargeted circularly permuted yellow fluorescent protein (mt-cpYFP) cDNA (total volume $10 \mu \mathrm{l}$ in $71 \mathrm{mM} \mathrm{NaCl}$ ) using a 30-gauge needle. The footpad was then electroporated $(100 \mathrm{~V} / \mathrm{cm}, 20-\mathrm{ms}$ duration, 20 pulses delivered at 1 $\mathrm{Hz}$ ) using subcutaneous gold-plated electrodes placed perpendicular to the long axis of the muscle. Individual FDB fibers were isolated by enzymatic dissociation 1 week after electroporation as described previously [42].

\section{mSOF measurement and analyses}

Acutely isolated FDB fibers (from $n=4$ to 5 mice) were plated on glass-bottom dishes in a rodent Ringer's solution $(146 \mathrm{mM} \mathrm{NaCl}, 5 \mathrm{mM} \mathrm{KCl}, 1 \mathrm{mM} \mathrm{MgCl}, 2 \mathrm{mM}$ $\mathrm{CaCl}_{2}, 10 \mathrm{mM}$ HEPES, pH 7.4) and then loaded with 10 $\mathrm{nM}$ tetramethylrhodamine ethyl ester (TMRE) to enable simultaneous monitoring of changes in mitochondrial membrane potential during mitoflash activity. Mt-cpYFP and TMRE were excited using 488-nm ( $8 \times$ attenuation) and 543-nm (64× attenuation) lasers and detected at 515/30-nm and 605/75-nm emission, respectively. Timelapse $x, y$ images were acquired at $1.24 \mathrm{~s} /$ frame and $512 \times 512$ resolution, with a total of 100 frames, using a Nikon Eclipse C1 Plus Confocal microscope equipped with a SuperFluor 60x (1.4 NA) oil-immersion objective. Automated detection and analysis of individual mitochondrial superoxide flash (mSOF) events during timelapse $x, y$ imaging was performed using 'Flash Collector,' a MATLAB-based image analysis program described in detail previously [42]. Flash frequency, amplitude, and full duration at half maximal amplitude (FDHM) were expressed as the number of events/1,000 $\mu^{2} \cdot 100 \mathrm{~s}, \Delta F /$ $F_{0}$, and seconds, respectively. Output data from Flash Collector were tabulated, averaged, and evaluated for 
statistical significance using Microsoft Excel and SigmaPlot software suites.

\section{Glutathione assay}

Control and NAC-treated (for 2 months) 4-month-old WT and CASQ1-null mice were sacrificed and hind limb muscles removed. Hind limb muscles were homogenized, and total glutathione and oxidized glutathione (GSSG) levels were measured according to [43]. Briefly, $0.1 \mathrm{~g}$ of tissue sample was homogenized in $1 \mathrm{ml}$ of fresh extraction buffer $(0.1 \%$ Triton X-100 and $0.6 \%$ sulfosalicylic acid in $0.1 \mathrm{M}$ potassium phosphate buffer with 5 $\mathrm{mM}$ EDTA disodium salt, $\mathrm{pH}$ 7.5) on ice three times using an MICCRA D-1 Homogenizer (ART Prozess- \& Labortechnik GmbH \& Co., Müllheim, Germany). After homogenization, 5\% trichloroacetic acid was added to the solution, and the homogenized tissue sample was centrifuged at $8,000 \times g$ for $10 \mathrm{~min}$ at $2^{\circ} \mathrm{C}$ to $4^{\circ} \mathrm{C}$ to obtain a supernatant containing glutathione. The sample was then stored at $-80^{\circ} \mathrm{C}$ until further use. The assay used for measurement of total glutathione and GSSG is based on the reaction of reduced glutathione (GSH; directly or through conversion of GSSG to GSH by 2vinylpyridine) with $5,5^{\prime}$-dithio-bis (2-nitrobenzoic acid) (DTNB), which produces the 5-thio-2-nitrobenzene (TNB) chromophore measured at $412 \mathrm{~nm}$. The rate of TNB formation (change in absorbance $\mathrm{min}^{-1}$ ) is proportional to the concentration of GSH in the sample. The assay was performed in 96 well plates (96 Well Tissue Culture Testplate; Spl Life Sciences, Gyeonggi-do, Korea) using an Absorbance Microplate Reader SpectraMAX 190 (Molecular Devices, Sunnyvale, CA, USA). The GSH concentration in each sample was determined by linear regression using a standardized GSH curve. The GSH/GSSG ratio was then calculated.

\section{Statistical analyses}

Statistical significance for measurements of cores in WT and CASQ1-null mice was evaluated using a two-tailed Fisher exact test. Two-tailed Student's unpaired $t$ test or one-way ANOVA test was used for statistical analysis of all other experiments as appropriate. In all cases, statistical significance was set at either $P<0.05\left(^{*}\right)$ or $P<0.01$ $(* *)$. Statistical analyses were performed using GraphPad Prism 5 (GraphPad Software, San Diego, CA, USA). All measurements were expressed as mean \pm SEM.

\section{Results}

Due to the high mortality of CASQ1-null male mice, all experiments in this study were conducted using female WT and CASQ1-null animals. See the 'CASQ1-null mice' subsection of the 'Methods' section and Additional file 1: Figure S1 for additional details.
EDL muscle from CASQ1-null mice exhibit decreased force output and slowed kinetics of contraction

To determine the functional output of muscle from CASQ1-null mice at different ages, we performed grip strength measurements. CASQ1-null mice exhibited significantly reduced normalized grip strength $(\mathrm{mN} / \mathrm{g})$ compared to that of WT mice across all ages tested

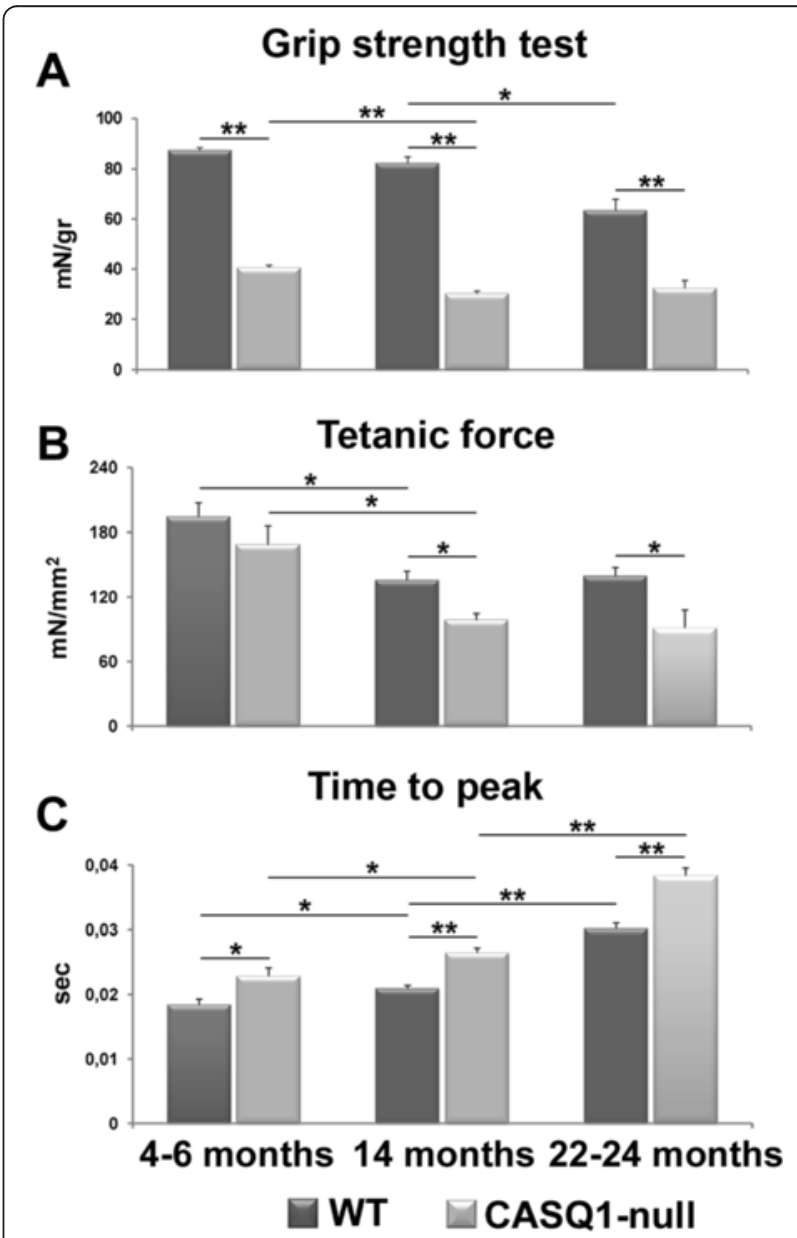

Figure $1 \mathrm{EDL}$ muscles from CASQ1-null mice exhibit impaired force output and slowed kinetics of contraction. (A) Maximal grip strength (normalized to body weight) is significantly reduced across all ages in CASQ1-null mice compared to WT. (B) Peak-specific force measured during maximal fused isometric tetanic contractions in excised EDL muscles is significantly reduced in 14- and 22- to 24-month-old CASQ1-null mice compared to WT and in CASQ1-null mice from 4 to 6 months to 14 months of age. (C) Time-to-peak isometric twitch force is significantly increased in CASQ1-null mice across all ages compared to WT and increases both in WT and CASQ1-null mice up to 22 to 24 months. Number of animals tested for grip strength test: WT, $n=175,31$, and 9 for 4 to 6,14 , and 22 to 24 months of age, respectively; CASQ1-null, $n=157,51$, and 9 for 4 to 6,14 , and 22 to 24 months of age, respectively. Number of animals tested for force and contraction kinetics: WT, $n=5,8$, and 10 for 4 to 6,14 , and 22 to 24 months of age, respectively; CASQ1-null, $n=5,5$, and 6 for 4 to 6,14 , and 22 to 24 months of age, respectively. Data are given as means \pm SEM $\left(* P<0.05,{ }^{*} P<0.01\right)$. EDL, extensor digitorum brevis; CASQ1, skeletal isoform of calsequestrin; $W T$, wild type. 
(Figure 1A), consistent with impaired neuromuscular function.

Interestingly, while grip strength in CASQ1-null mice decreased significantly between 4 to 6 and 14 months, the deficit remained stable from 14 months to 22 to 24 months. The central role of skeletal muscle in this decrease in force output was confirmed by ex vivo experiments in isolated EDL muscles (Figure 1B,C). Specifically, maximal tetanic-specific force (measured at optimal length at $120 \mathrm{~Hz}$ under isometric conditions) was significantly $(P<0.05)$ reduced in CASQ1-null mice compared to WT mice at both 14 and 22 to 24 months of age, but not at 4 to 6 months of age (Figure 1B). Again, as in the grip strength test above, peak tetanic force significantly $(P<0.05)$ decreased in CASQ1-null mice from 4 to 6 months to 14 months but remained unchanged from 14 months to 22 to 24 months. Finally, time-to-peak tetanic contraction was also significantly increased in EDL muscles from CASQ1-null mice compared to WT mice across all evaluated ages (Figure 1C; $P<0.05$ and $P<0.01)$. Time to peak in CASQ1-null EDLs becomes progressively slower up to 22 to 24 months.

\section{Formation of cores in EDL muscle fibers of CASQ1-null mice}

To provide insight into the reduced contractility of EDL muscles from CASQ1-null mice (Figure 1), we used a combination of light and electron microscopy (EM) to compare the ultrastructure of muscle fibers of EDL muscles from age-matched WT and CASQ1-null mice. While significant structural modifications were rare at 4 to 6 months of age, the percentage of EDL fibers from CASQ1-null mice exhibiting extended areas of morphological alterations significantly increased from 4 to 6 months to 14 months and older (Figures 2, 3, and 4 and Additional file 3: Table S1). At the EM level, the structural alterations observed in fibers from CASQ1-null mice reflected a progression from modest to more severe irregularities, often characterized by the presence of swollen mitochondria (Figure 2A, arrowheads and inset) and misalignment of contractile elements (Figure 2B, arrows), some Z-line streaming (Figure 2C, empty arrows), and formation of regions of myofibril contractures (Figure 2D). Similar areas of disrupted cross-striations and/or hyper-contracture were only very rarely observed in EDL fibers of WT mice (Additional file 3: Table S1), even at the most advanced age investigated (27 months).

Although structural alterations observed in muscle fibers from CASQ1-null mice were variable in appearance, we classified two primary forms of disarray detectable by both light and electron microscopy: 1) extensive areas lacking regular cross-striation and order, termed unstructured cores (Figure 2B,C; and Figure 3C, stars), and 2) areas exhibiting extreme sarcomere shortening, termed contracture cores (Figure 2D; and Figure 3E,F, empty arrows). This terminology was previously introduced by Boncompagni et al. [35] in a mouse model carrying a mutation in RYR1 that results in $\mathrm{MH}$ with cores in humans. Such alterations were also visible by immunolabeling with anti-RYR1 antibodies, which revealed a partial disruption in the normally highly ordered double-row pattern of CRU distribution within regions of unstructured cores (compare Figure 3B,D, white boxes and insets).
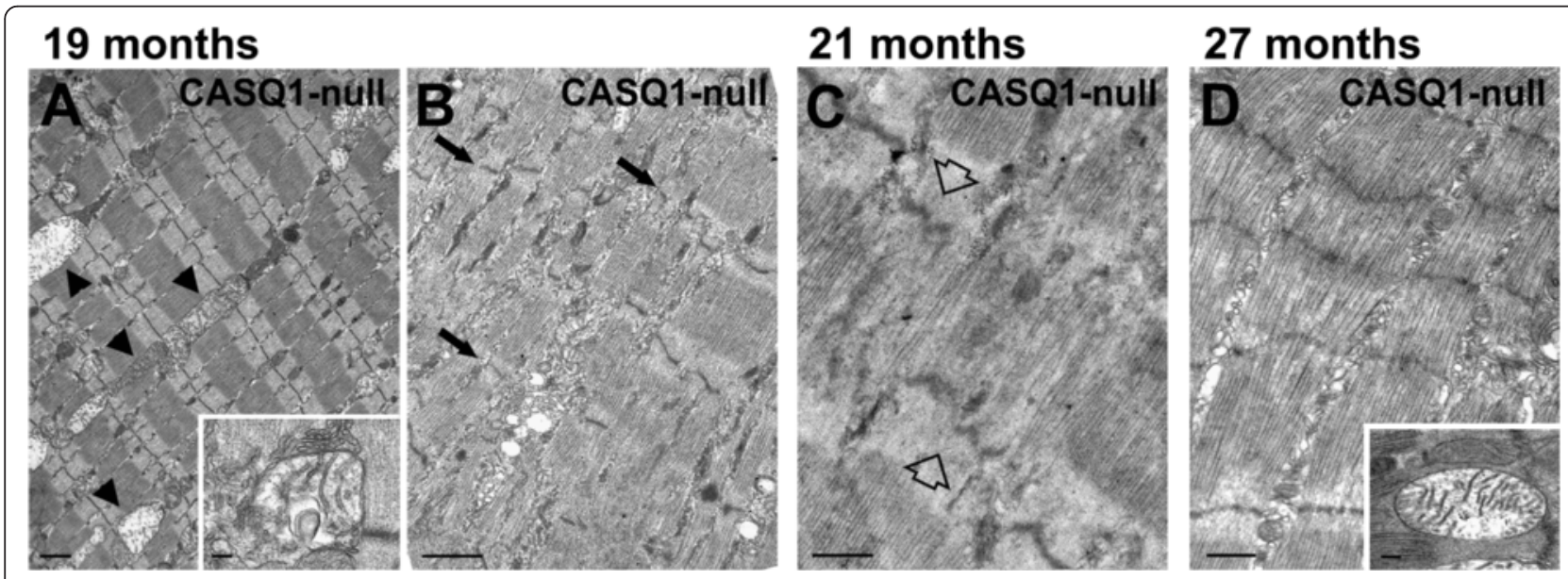

Figure 2 Structural abnormalities in EDL muscle fibers from aged CASQ1-null mice. (A) Many fibers (even at 19 months of age) do not present severe disarrangement of contractile elements. Nevertheless, even in these fibers, normal muscle ultrastructure is not well preserved, with partial misalignment of myofibrils and frequent mitochondria exhibiting clear signs of structural damage/swelling (arrowheads, see also inset). (B, C) Unstructured cores, areas in which Z lines ((B), small arrows) and contractile elements are out of register and sarcomeres are partially disarranged, are frequently observed in fibers from EDL muscle from CASQ1-null mice. Z-line streaming is also present in some fibers ((C), empty arrows). (D) Contracture cores, areas of excessive sarcomere shortening, contain fewer and severely damaged mitochondria (inset). See Figure 1 and Additional file 3: Table S1 for detailed quantitative analyses. Scale bars: (A, B), $1 \mu \mathrm{m}$; (C, D), $0.5 \mu \mathrm{m}$; insets, $0.1 \mu \mathrm{m}$. EDL, extensor digitorum brevis; CASQ1, skeletal isoform of calsequestrin. 


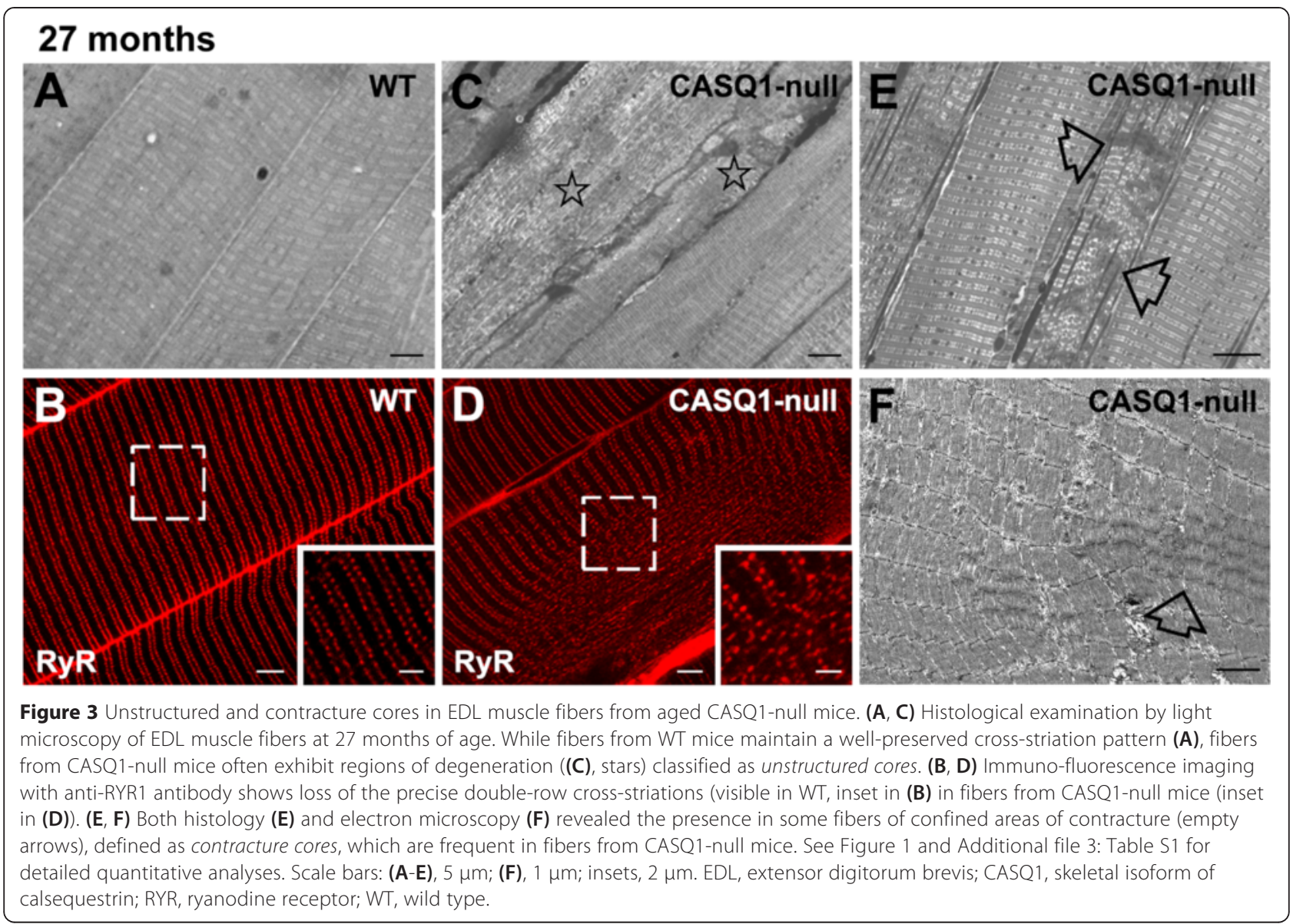

We quantified the percentage of fibers presenting either unstructured or contracture cores (Figure 4 and Additional file 3: Table S1). While fibers with extended structural alterations in CASQ1-null mice were found in only $2 \%$ of fibers at 4 months of age, their frequency increased significantly from 4 to 14 months of age (about $25 \%$ ), with no further significant changes at older ages (Figure 4 and Additional file 3: Table S1, column C). However, these regions typically occupied only a limited area at early ages (14 months old), becoming much more extensive at older ages (20 to 27 months old). Fibers presenting cores were very rare in WT muscle even at the oldest age analyzed (only $8 \%$ at 27 months of age), and areas of alteration were usually very confined. Statistical analyses (Figure 4 and Additional file 3: Table S1) indicate that the total percentage of fibers presenting alterations in EDL muscles of CASQ1-null mice was significantly higher than that observed in WT mice at each of the time points analyzed except at 4 months (Figure 4).

Reduced CSA of EDL muscle fibers from CASQ1-null mice Female CASQ1-null mice exhibited a significant reduction (approximately 15\%) in body weight across all ages compared to age-matched WT female mice (Additional file 4: Table S2), similar to that reported previously for adult male mice [25]. As average EDL dry weight and muscle/body weight ratio were reduced approximately $20 \%$ to $30 \%$ in CASQ1-null mice across all ages (Additional file 4: Table S2), the decrease in total body weight is likely, or at least in part, due to the reduction in muscle mass. Indeed, measurements of CSA of EDL muscle fibers at three different ages (Figure 5) verified that fiber size was significantly reduced in EDL muscles from CASQ1-null mice compared to the age-matched WT mice across all ages $(P<0.01)$. Average CSA decreased from 4 to 6 months to 24 months of age in both genotypes (33.3\% in CASQ1-null mice vs. $41.3 \%$ of WT fibers). Interestingly, the major drop in CSA in fibers from CASQ1-null mice occurred between 4 and 14 months of age, with no further significant change observed from 14 to 24 months (Figure 5), a finding that parallels the functional (Figure 1A,B) and structural (Figure 4 and Additional file 3: Table S1) data. Microarray analyses of EDL muscles from 4-month-old CASQ1-null mice revealed a significant up-regulation of four atrogenes (CathepsinL, Bnip3, Psmd1, and Atrogin1) that belong to the autophagy and ubiquitin-proteasome pathways, 


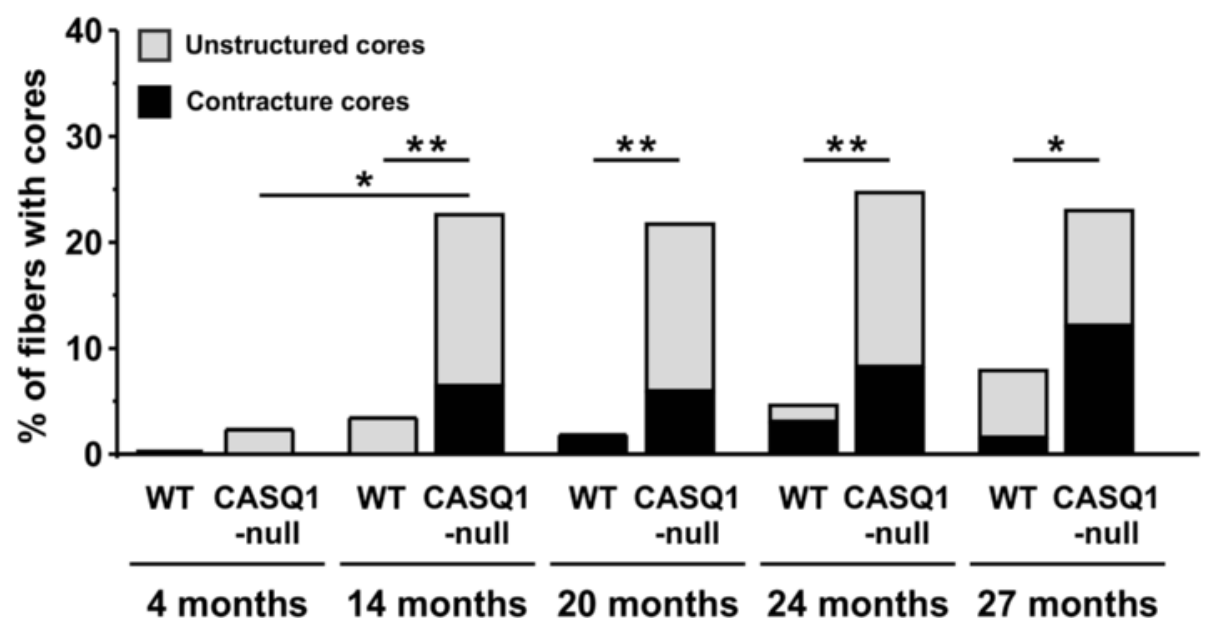

Figure 4 Increased incidence of unstructured and contracture cores in 14- to 27-month-old CASQ1-null mice. While the percentage of fibers in WT mice exhibiting structural cores remained low across all ages (maximum of $8 \%$ at 27 months of age), muscle fibers from CASQ1-null mice exhibited a significantly increased frequency $\left({ }^{*} P<0.05\right)$ of structural cores from 4 months of age (approximately $\left.2 \%\right)$ to 14 months $(23 \%)$, remaining approximately unchanged thereafter (see also Additional file 3: Table S1 for additional detail). CASQ1, skeletal isoform of calsequestrin; WT, wild type.

suggesting one possible mechanism for the increased atrophy observed in muscle fibers from CASQ1-null mice (Additional file 5: Table S3).

Age-dependent increase in mitochondrial damage in EDL muscle from CASQ1-null mice

Muscle fibers from young (4- to 6-month-old) CASQ1null mice do not exhibit structural cores (though clearly visible at later stages (see Figures 2, 3, and 4 and Additional file 3: Table S1). However, structural analyses at higher magnification with EM of muscle fibers at this age revealed that a) the frequency of mitochondria in muscle fibers from CASQ1-null mice is increased (Figure 6A,B, empty arrows), an observation consistent with previous findings [25], and b) mitochondria exhibiting evidence of structural damage were more frequently observed in muscle fibers from CASQ1null mice (Figure 6B, black arrows and Figure 6G).

We measured relative mitochondrial volume (expressed as a percentage of total fiber volume) in EDL muscle from 4- to 6-month-old mice and confirmed a significant increase (approximately 2.4-fold) in cellular mitochondrial volume in muscle fibers from 4- to 6-month-old CASQ1null mice compared to age-matched WT mice (Figure $6 \mathrm{C}$; $P<0.01)$. As the transcriptional co-activator PGC- $1 \alpha$ regulates many aspects of oxidative metabolism [44], including mitochondrial biogenesis and fiber-type switching from glycolytic toward more oxidative fibers [45], we quantified PGC-1 $\alpha$ expression by Western blot analysis in EDL muscle homogenates from 4-month-old WT and CASQ1-null mice (Additional file 2: Figure S2). PGC-1 $\alpha$ levels (Additional file 2: Figure S2, panel A, lower band pointed by the arrow) were increased almost twofold in EDL muscle homogenates from adult CASQ1-null mice $(P<0.01)$. This significant increase in $P G C-1 \alpha$ suggests that the observed increase in mitochondrial volume in muscle from CASQ1-null mice is due in part from increased mitochondrial biogenesis. In addition, consistent with increased oxidative fiber content (including increased capillarization, as well as mitochondrial, myoglobin, and heme content), EDL muscles from CASQ1-null mice appear more red-colored than EDL muscles from WT mice (see Additional file 6: Figure S3).

We also quantified the number of mitochondria exhibiting one or more of the following alterations: a) swelling, b) presence of vacuoles, c) clear disruption of the external membrane and/or internal cristae, and d) myelin figures (see examples in Figure 6D,E,F). Although the frequency of damaged mitochondria varied significantly from fiber to fiber and from area to area, the percentage was significantly $(P<0.01)$ increased in muscle fibers from 4- 6-month-old CASQ1-null mice compared to that of age-matched WT mice $(15.08 \% \pm 0.9 \%$ vs. $2.65 \% \pm 0.5 \%$, respectively; Figure $6 \mathrm{G}$ and Table 1). Interestingly, PGC- $1 \alpha$ expression is slightly down-regulated in CASQ1null muscle at 25 months of age (see Additional file 2: Figure S2), suggesting that the replacement of damaged mitochondria in the muscle of aged CASQ1-null mice could be impaired, thus resulting in an accumulation of damaged mitochondria.

As detailed above, muscle fibers from CASQ1-null mice exhibited an age-dependent increase in the development of structural cores (Figures 2, 3, 4 and Additional file 3: Table S1). In order to correlate mitochondrial damage to 


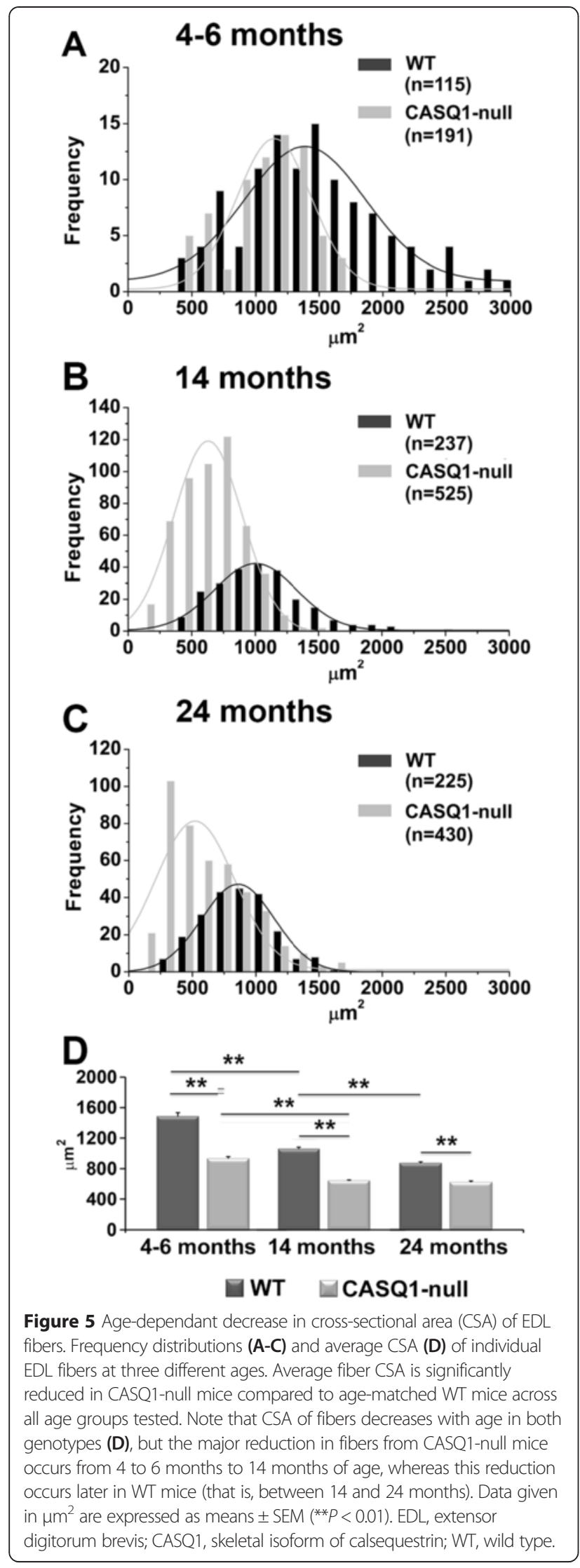

core formation, we measured the relative volume occupied by mitochondria in non-core and core regions of aged (27 months old) CASQ1-null mice. In regions of muscle fibers exhibiting only modest alterations (similar-normal areas), mitochondrial volume was increased in aged mice compared to young adult mice $(13.26 \% \pm 0.86 \%$ vs. $8.21 \% \pm 0.29 \%$ at 27 months and 4 to 6 months, respectively; Figure 6C), consistent with the visual impression of increased mitochondrial size with age (Figure 2A). Interestingly, mitochondrial volume was significantly reduced $(6.01 \% \pm 0.52 \%, P<$ 0.01 ) within unstructured and contracture cores (areas such as those in Figure 2B,C,D), consistent with loss of mitochondria in core regions.

\section{Increased oxidative stress and mSOF activity in muscle from CASQ1-null mice}

The delicate balance between the production of oxidative species and antioxidant defense is severely compromised in several pathological conditions [46]. Given that mitochondria are a primary source of reactive oxygen species (ROS) generation and mitochondrial number/volume are increased in muscle fibers of CASQ1null mice (Figure 6), we compared the overall redox state (GSH and GSSG) and the temperature dependence of quantal mitochondrial superoxide production (termed mitochondrial superoxide flashes or mSOFs $[42,47])$ in skeletal muscle from 4- to 6-month-old WT and CASQ1-null mice (Figure 7). This age was chosen for these analyses because it represents a time at which mitochondrial changes have already occurred but is prior to the development of unstructured and contraction cores. The GSH/GSSG ratio was significantly $(P<$ 0.01) reduced in EDL muscle homogenates from CASQ1null mice compared to that of WT mice (Figure 7A), consistent with increased levels of oxidative stress in muscle of CASQ1-null mice. Second, we determined the frequency and amplitude (Figure 7B,C) of transient bursts of mitochondrial superoxide production that occur simultaneously with mitochondrial depolarization in quiescent FDB fibers from 4- to 5-month-old WT (Figure 7D,E,F) and CASQ1null mice (Figure 7G,H,I). Whereas no significant differences in mSOF frequency or amplitude were detected at room temperature $\left(20^{\circ} \mathrm{C}\right)$, a significant $(P<0.05)$ increase in $\mathrm{mSOF}$ frequency was observed at physiological temperature $\left(37^{\circ} \mathrm{C}\right)$ in FDB fibers from CASQ1-null mice $\left(17.7 \pm 1.3\right.$ and $25.7 \pm 1.3$ flashes $/ 1,000 \mu \mathrm{m}^{2} \cdot 100 \mathrm{~s}$ at $20^{\circ} \mathrm{C}$ and $37^{\circ} \mathrm{C}$, respectively) (Figure $7 \mathrm{~B}, \mathrm{C}$ ). This temperaturedependent increase was not observed in FDB fibers from WT mice: $17.1 \pm 1.1$ and $19.2 \pm 1.2$ flashes $/ 1,000 \mu \mathrm{m}^{2} \cdot 100 \mathrm{~s}$ at $20^{\circ} \mathrm{C}$ and $37^{\circ} \mathrm{C}$, respectively. Importantly, the significant $(P<0.05)$ increase in both $\mathrm{mSOF}$ frequency (Figure $7 \mathrm{~B}$ ) and amplitude (Figure $7 \mathrm{C}$ ) at $37^{\circ} \mathrm{C}$ in fibers from CASQ1null mice is consistent with the increased oxidative stress 


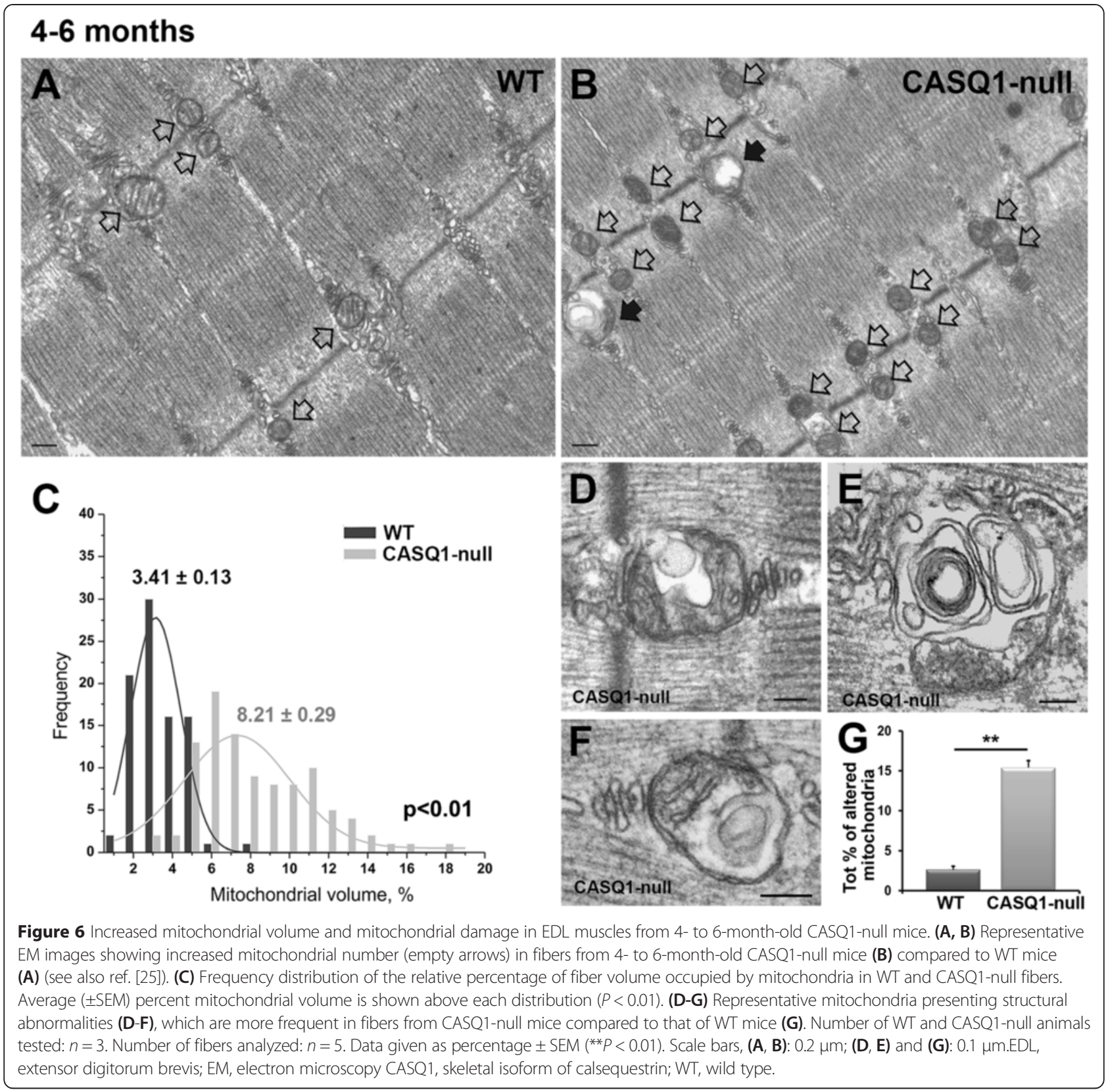

Table 1 Percentage of mitochondria exhibiting different types of structural abnormalities in WT and CASQ1-null animals

\begin{tabular}{llllll}
\hline EDL (4 to $\mathbf{6}$ months) & \multicolumn{2}{c}{ Mitochondrial alterations (\%) } & \multicolumn{2}{c}{$\begin{array}{l}\text { Total \% of altered } \\
\text { mitochondria }\end{array}$} \\
\cline { 2 - 5 } & $\begin{array}{l}\text { Swollen } \\
\text { mitochondria }\end{array}$ & $\begin{array}{l}\text { Containing } \\
\text { vacuole }\end{array}$ & $\begin{array}{l}\text { Disruption of ext. } \\
\text { membr./int. cristae }\end{array}$ & $\begin{array}{l}\text { Containing } \\
\text { myelin figures }\end{array}$ & 0.16 \\
\hline WT & 1.45 & 0.80 & 0.24 & $1.23^{* *}$ & $2.65 \pm 0.5$ \\
CASQ1-null & $6.22^{* *}$ & $5.66^{* *}$ & $1.96^{* *}$ & $15.08 \pm 0.9^{* *}$ \\
\hline
\end{tabular}

In EDL muscles from 4- to 6-month-old mice, the percentage of mitochondria exhibiting one or more types of structural abnormality (swollen, disrupted internal cristae, vacuoles, or myelin figures) is significantly increased in CASQ1-null mice. Data are means \pm SEM $(* * P 0.01)$. CASQ1, skeletal isoform of calsequestrin; WT, wild type. 


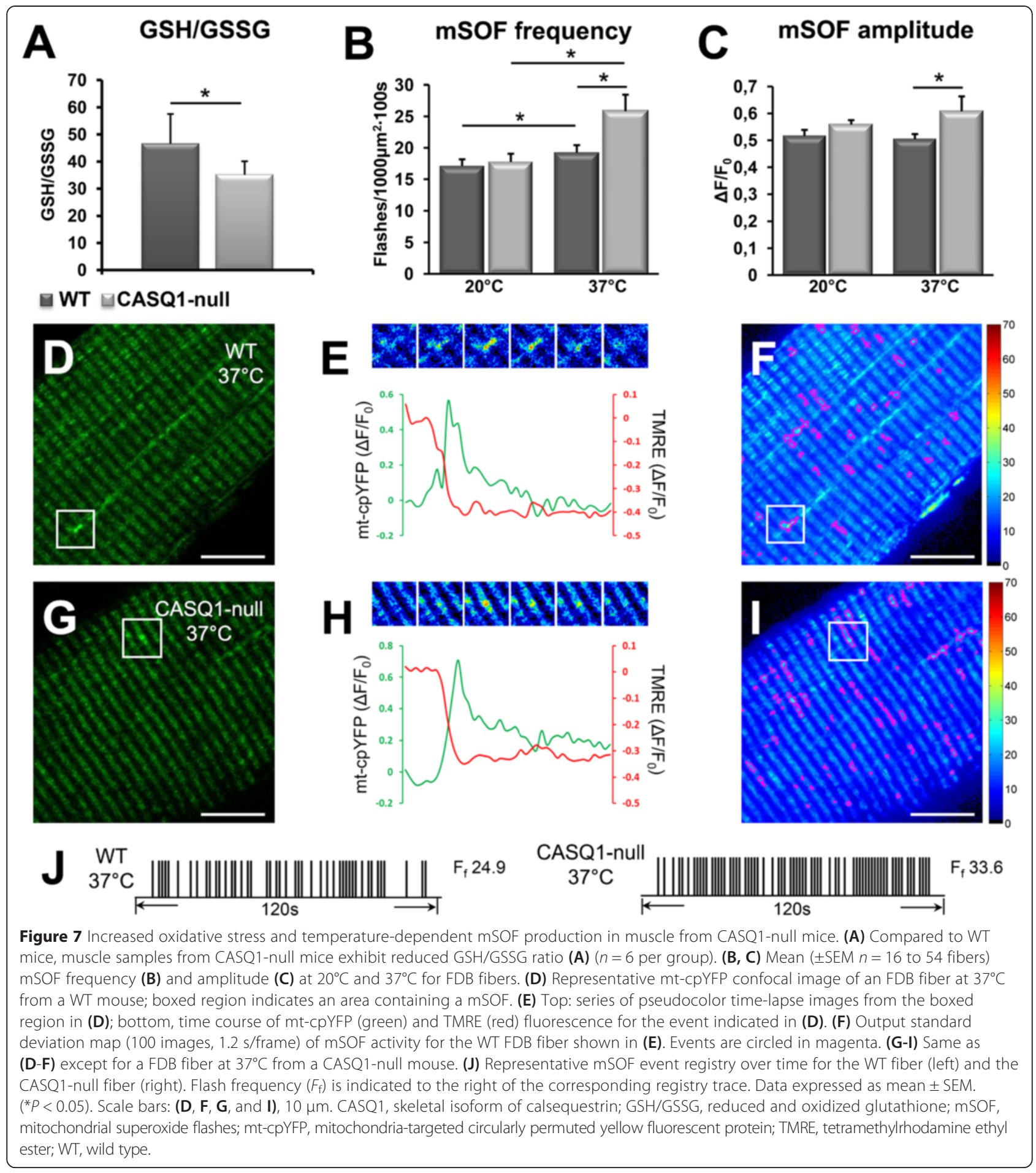

observed in hind limb muscles from CASQ1-deficient mice (Figure 7A). As elevated levels of ROS can damage critical cellular components including proteins and membrane lipids [48], these results may explain the increased mitochondrial damage observed in muscle fibers from 4- to 6-month-old CASQ1-null mice (Figure 6G).
NAC treatment of CASQ1-null mice reduces oxidative stress and mSOF production, prevents mitochondrial volume/ damage, and improves muscle function

To determine if excessive oxidative stress in CASQ1-null mice is responsible for mitochondrial alterations (Figure 6), muscle damage (Figures 2, 3, and 4), and reduced contractility (Figure 1), mice were provided NAC (a potent 
anti-oxidant and precursor for glutathione synthesis) in their drinking water for 2 months (that is, from 2 to 4 months of age). Long-term NAC treatment in-vivo normalized muscle GSH/GSSG to control levels (Figure 8A; $P<0.05)$. In addition, acute in-vitro treatment of isolated FDB fibers with $2.5 \mathrm{mM} \mathrm{NAC}$ for $30 \mathrm{~min}$ reduced $\mathrm{mSOF}$ frequency at $20^{\circ} \mathrm{C}$ and $37^{\circ} \mathrm{C}$ in both WT and CASQ1-null mice. Specifically, NAC treatment normalized $\mathrm{mSOF}$ frequency at $37^{\circ} \mathrm{C}$ in fibers from CASQ1-null mice to a level not different to that of fibers from WT mice in the absence of NAC treatment (Figure $8 \mathrm{~B}$, dash line; $P<$ 0.05). No change in $\mathrm{mSOF}$ amplitude was detected (see Additional file 7: Figure S4). Together, results in Figure 8A,B indicate that NAC treatment restored the oxidative balance muscle from CASQ1-null mice. Importantly, long-term NAC treatment of CASQ1-null mice markedly reduced structural alterations typically observed at 4 months of age, including increased mitochondrial volume (Figure $8 \mathrm{C} ; P<0.05$ and $P<0.01$ ) and mitochondria damage (Figure 8D; $P<0.01$ ). Finally, NAC treatment also partially rescued overall neuromuscular function, as shown by a significant improvement in grip strength (Figure 8E; $P<0.01$ ).

\section{Discussion}

In humans, CCD and MHS are related disorders as some patients with CCD also test positive for MHS $[10,16]$. Thus, individuals with CCD are typically considered at risk for an $\mathrm{MH}$ episode during anesthesia using triggering agents (for example, halothane). In this regard, we previously reported that CASQ1-null mice are susceptible to lethal halothane- and heat-induced hypermetabolic episodes $[29,31]$, a phenotype that closely resembles MHS in
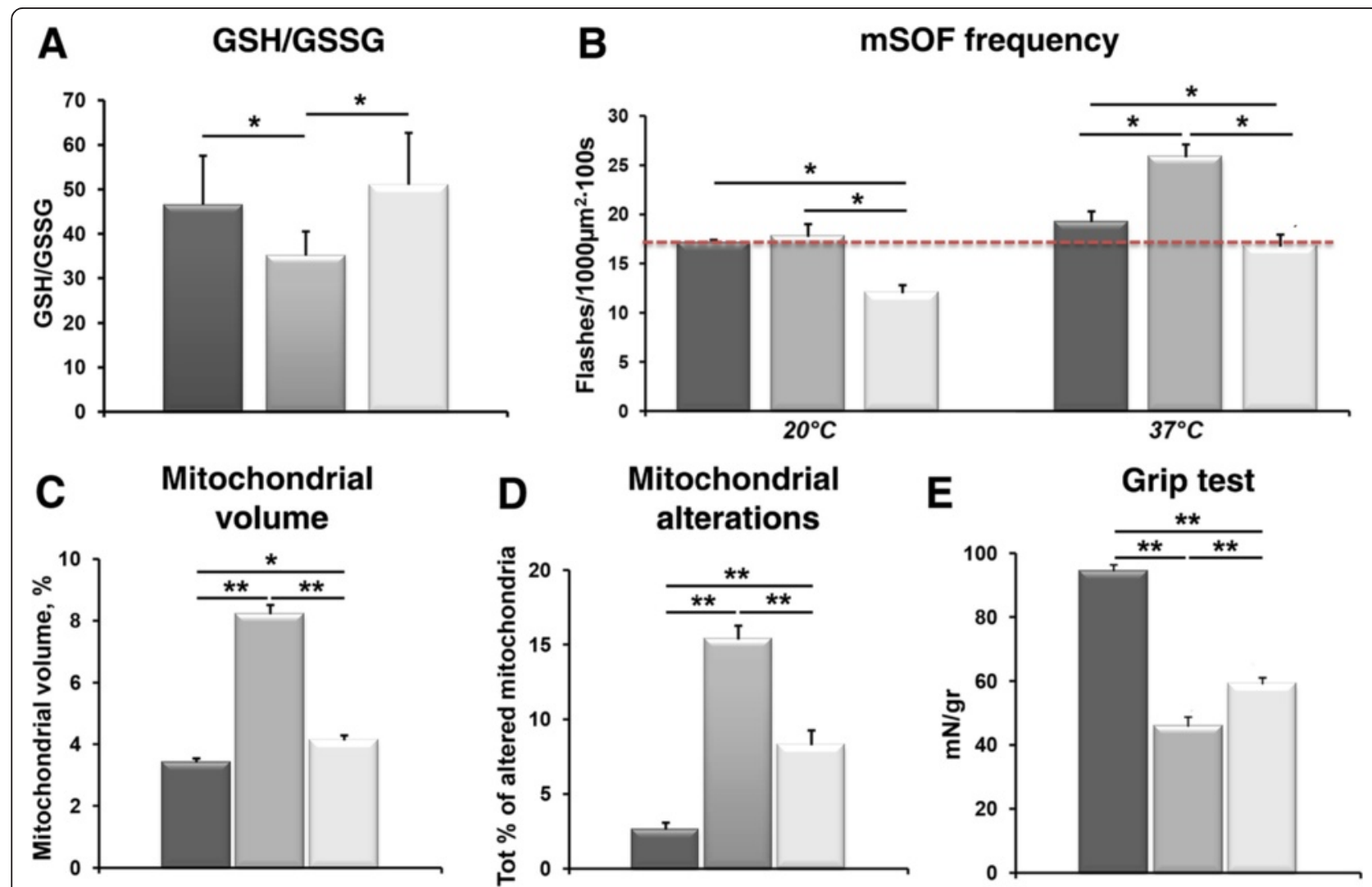

WT $\square$ CASQ1-null $\square$ CASQ1-null+NAC

Figure $8 \mathrm{~N}$-acetylcysteine (NAC) reduces oxidative stress, mSOF production, and mitochondrial volume/damage and improves muscle function. (A) GSH/GSSG ratio was restored to normal levels in muscle homogenates from CASQ1-null mice treated for 2 months with NAC ( $n=6$ per group). (B) Acute NAC treatment in FDB fibers reduced mSOF frequency at both $20^{\circ} \mathrm{C}$ and $37^{\circ} \mathrm{C}$. (C-E) NAC treatment of CASQ1-null mice for 2 months reduced total mitochondrial volume (C) and mitochondrial damage (D) and improved grip strength (E). Number of WT animals (without NAC treatment) and of old CASQ1-null animals tested: $n=3$. Number of fibers analyzed: $n=5$. Number of adult CASQ1-null animals (after NAC treatment) tested: $n=3$. Number of analyzed fibers: $n=5$. Data expressed as mean \pm SEM $\left({ }^{*} P<0.05\right.$ and $\left.{ }^{* *} P<0.01\right)$. CASQ1, skeletal isoform of calsequestrin; GSH/GSSG, reduced and oxidized glutathione; FDB, flexor digitorum brevis; mSOF, mitochondrial superoxide flashes; WT, wild type. 
humans and mouse models expressing human RYR1 mutations $[36,49]$.

The results presented in this study reinforce the concept that the pathophysiology of $\mathrm{MH}$ and CCD share a common underlying mechanism. Specifically, we demonstrate that CASQ1-null mice develop a myopathy characterized by mitochondrial damage at early ages that precede the development of structural and contracture cores at later ages (Figures 2, 3, and 4 and Additional file 3: Table S1). Similar alterations were described previously in biopsies from patients diagnosed with CCD and in RYR1 knock-in mouse models of core myopathy $[35,50]$. We also investigated the mechanisms that contribute to the mitochondrial damage observed in CASQ1-null mice and identified excessive production of ROS as a key early event in the myopathic cascade (possibly due to increased mitochondrial content and damage, see the 'CASQ1-null muscle shift toward a more oxidative phenotype' section below), confirming previous findings in a different mouse model of CCD $[35,49]$. The common molecular mechanism in these mouse models could be excessive SR leak (see the 'Proposed pathogenic mechanism for mitochondrial damage and myopathy in CASQ1-null mice' section below), resulting either directly by the mutations in the SR $\mathrm{Ca}^{2+}$ release channel (in RYR1 ${ }^{\mathrm{Y} 522 \mathrm{~S} / \mathrm{WT}}$ knock-in mice) $[36,49]$ or indirectly by the loss of CASQ1-mediated inhibition of RYR1 channel activity in CASQ1-null mice [29,31]. Importantly, we demonstrated a central role for increased oxidative stress in the mitochondrial damage and myopathy by rescuing multiple phenotypes in CASQ1-null mice with anti-oxidant treatment (Figure 8). Whereas oxidative stress, mitochondrial damage, or core formation may definitely contribute to reduce muscle strength reported in Figure 1, we cannot rule out the possibility that the impaired force output observed in CASQ1 KO muscle is still primarily due to the altered SR $\mathrm{Ca}^{2+}$ buffer and content and reduced $\mathrm{Ca}^{2+}$ release from RYRs [25-28].

\section{CASQ1-null muscle shift toward a more oxidative phenotype}

Whereas fiber-type switching (that is, change of myosin isoform expression) does not occur in CASQ1-null mice [25], the increase in mitochondrial number [25] and volume (Figure 6) in EDL muscle of CASQ1-null mice is consistent with a shift toward increased oxidative metabolism. Consistent with this, EDL muscles from CASQ1-null mice are more red-colored than those from age-matched WT mice (Additional file 6: Figure S3). The increased mitochondrial density observed in EDL muscle fibers (Figure 6; [18]) may be due to the observed increased in PGC- $1 \alpha$ expression (Additional file 2: Figure S2), a transcriptional co-activator that stimulates mitochondrial biogenesis and promotes the remodeling of muscle tissue toward increased oxidative metabolism $[45,51]$. The exact molecular signals linking CASQ1 deficiency to an up-regulation of PGC-1 $\alpha$ remain unclear and, thus, are worthy of further investigation. However, increases in cytoplasmic $\mathrm{Ca}^{2+}$ and metabolic demand, as well as ATP deficiency, are all known to enhance PGC- $1 \alpha$ expression [52-54]. Thus, since free myoplasmic $\mathrm{Ca}^{2+}$ levels are increased at physiological temperature in CASQ1-null fibers [29], a $\mathrm{Ca}^{2+}$-mediated increase in PGC-1 $\alpha$ expression could underlie the up-regulation of this signaling pathway.

\section{Increased oxidative stress in CASQ1-null mice}

A significant fraction of mitochondria in muscle from CASQ1-null mice exhibit morphological abnormalities (Figures 2 and 6). This increased structural damage of mitochondria (Figure 6) could result from the combined effects of elevated myoplasmic $\mathrm{Ca}^{2+}$ [29] and redox imbalance. Indeed, mitochondria, which are located in proximity of CRUs $[25,55]$, are in a position to be directly impacted by $\mathrm{Ca}^{2+}$ disturbances at adjacent CRUs that lack CASQ1. In addition, the decrease in GSH/ GSSG ratio and increase in mSOF activity (Figure 7) in muscle from CASQ1-null mice are indicative of increased mitochondrial-derived oxidative stress. Mitochondria, which produce superoxide as a byproduct of oxygen consumption due to electron slippage from the electron transport chain during aerobic respiration [56], are a primary source of ROS production within the cell. However, our results do not preclude a potential role of additional ROS sources, including NADPH oxidase [57], to the increased oxidative stress observed in muscle from CASQ1-null mice. Nevertheless, the increase in mitochondrial number/volume (Figure 6) [29] and enhanced mitochondrial superoxide production (Figure 7) likely contribute to the augmented oxidative stress observed in muscle from CASQ1-null mice (Figure 7A). These changes could in turn lead to oxidative modifications/damage to critical cellular proteins, membrane lipids, and various organelles, including mitochondria [48]. Furthermore, damaged mitochondria may not function properly and, thus, produce additional ROS, driving a dangerous feed-forward mechanism that further exacerbates cellular damage. This vicious cycle resembles that described previously in a mouse model of $\mathrm{MH}$ with cores (RYR1 ${ }^{\text {Y522S/WT }}$ mice), which also exhibits a marked temperature-dependent increase in resting myoplasmic $\mathrm{Ca}^{2+}$, oxidative stress [49], and mSOF activity [42].

\section{Rescue by NAC treatment suggests a central role of oxidative stress in the myopathic cascade}

Enhanced oxidative stress plays a critical role in both $\mathrm{MH}$ susceptibility $[36,49]$ and the formation of cores that lack mitochondria in RYR1 ${ }^{\mathrm{Y} 522 \mathrm{~S} / \mathrm{WT}}$ mice [35]. A similar 
central role of increased oxidative stress in the mitochondrial damage and muscle dysfunction observed in CASQ1-null mice is supported by data presented in Figure 8. These studies demonstrate that treatment of CASQ1-null mice with a potent anti-oxidant (NAC) for 2 months restored the muscle redox state, reduced mitochondrial alterations, and improved muscle functionality (Figure 8). By analogy to that observed for RYR1 $1^{\mathrm{Y} 522 \mathrm{~S} / \mathrm{WT}}$ mice [49], we speculate that a reduction of oxidative stress in CASQ1-null mice may reduce S-nitrosylation of RYR1 to block the deleterious feed-forward cycle of increased $\mathrm{SR} \mathrm{Ca}^{2+}$ leak and ROS production. However, future experiments are needed to demonstrate a critical role for RYR1 $S$-nitrosylation in the mitochondrial proliferation, damage, and core myopathy observed in CASQ1-null mice.

\section{Proposed pathogenic mechanism for mitochondrial damage and myopathy in CASQ1-null mice}

The schematic in Figure 9 summarizes a proposed sequence of events that leads to mitochondrial proliferation, increased oxidative stress, and mitochondrial damage and, over time, the development of a core-like myopathy in CASQ1-null mice. Dainese et al. [29] reported that muscle fibers from CASQ1-null mice exhibit high-resting $\mathrm{Ca}^{2+}$ levels at body temperature. This excess in intracellular $\mathrm{Ca}^{2+}$ may result from enhanced SR $\mathrm{Ca}^{2+}$ leak (possibly due to loss of CASQ1mediated RYR1 inhibition) and/or excessive store depletion leading to store-operated $\mathrm{Ca}^{2+}$ entry (SOCE) from the extracellular space (step 1). Both effects are supported by existing literature: a) CASQ1 inhibits RYR1-opening probability and $\mathrm{Ca}^{2+}$ release [23,29], b) CASQ1-deficiency results in a temperature-dependent increase in resting free myoplasmic $\mathrm{Ca}^{2+}$ [58], and c) SOCE activation is increased in myotubes from CASQ1-null mice [58,59]. Indeed, enhanced SOCE could result from the greater susceptibility for activitydependent $\mathrm{SR} \mathrm{Ca}^{2+}$ depletion previously demonstrated in muscle fibers from CASQ1-null mice $[25,27,28]$. Enhanced resting $\mathrm{Ca}^{2+}$ levels would also increase ATP hydrolysis/demand required for SERCA-mediated SR $\mathrm{Ca}^{2}$ ${ }^{+}$reuptake (step 2), which together would increase PGC- $1 \alpha$ expression $[53,54]$ that promotes mitochondrial biogenesis and oxidative energy production (step 3). The ensuing increase in aerobic metabolism would lead to increased mitochondrial superoxide production (lowering GSH/GSSG ratio), which in turn would increase oxidative stress that further challenges mitochondrial integrity (steps 4 and 5) that promotes the formation of myopathic core-like regions (steps 6). Recent work supports a pivotal role for oxidative stress in altering the balance between protein synthesis and degradation in unloading and disuse atrophy [46].
Differences between structural modifications in muscle in CASQ1-null mice and those described in biopsies from patients carrying the p.Asp244Gly disease mutation in CASQ1

The first mutation in the CASQ1 gene linked to human disease was recently reported [34]. In this report, a dominant missense mutation in CASQ1 (N244G) was found in a group of patients with a vacuolar myopathy characterized by weakness, fatigue, and the presence of electron-dense inclusions. The structural modifications described in biopsies from these patients do not resemble in any way the alterations (that is, unstructured and contracture cores) described in CASQ1-null mice. However, the degree to which mitochondrial alterations are observed in muscle biopsies from CASQ1 N244G vacuolar myopathy patients is currently unknown. Importantly, large SR vacuoles containing electron-dense material, which likely reflects abnormal CASQ1 aggregation, are observed in patients with the N244G CASQ1 mutation. These alterations closely resemble those described previously in skeletal muscle fibers and cardiac myocytes from mice that overexpress CASQ1 and CASQ2 [60,61]. On the other hand, in muscle fibers from CASQ1-null mice, the SR is not swollen (but is actually reduced in size), CRUs form multiple junctions with T-tubules, and the terminal cisternae of the SR lacks electron dense material (that is, CASQ1) [25].

\section{Conclusions}

There are currently no curative treatments for CCD patients. Thus, a deeper mechanistic understanding of the molecular mechanisms that underlie mitochondrial damage and the formation of cores in CCD is needed in order to identify potential new therapeutic targets and, thus, to develop effective new interventions for this disorder. The mechanisms responsible for the loss of mitochondrial activity in core regions of muscle from CCD patients have also not yet been elucidated. Especially challenging is to explain how both gain- and loss-offunction mutations in RYR1 lead to myopathies characterized by damage and destruction of mitochondria (that is, $\mathrm{CCD}$ and multi-minicore disease), formation of structural and contracture cores, and muscle weakness.

Mutations in the CASQ1 gene have not been identified in patients diagnosed with either CCD or in MHS [32], and this represents a clear limit of our mouse model. Nevertheless, data of our study show that CASQ1 deficiency results in an age-dependent myopathy characterized by mitochondrial damage and formation of unstructured/contracture cores resembling those described in muscle biopsies from human CCD patients and in other mouse models of $\mathrm{MH}$ and CCD (see ref. [35,50]). In addition, we found that the molecular mechanisms observed in 


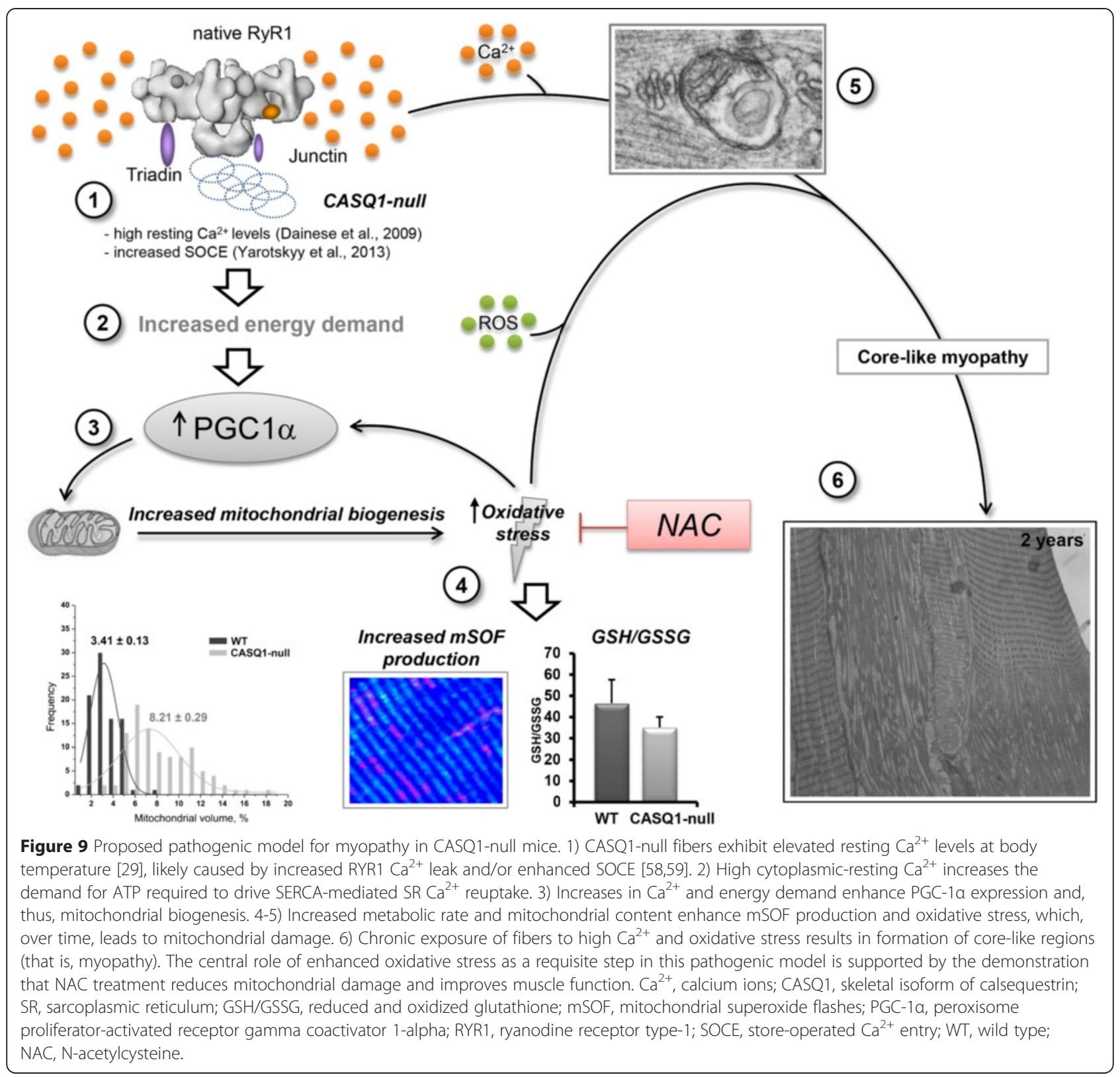

CASQ1-null fibers (that is, $\mathrm{Ca}^{2+}$ leak, excessive oxidative stress, mitochondrial damage) parallel those reported previously in a knock-in mouse carrying a human mutation [35].

We propose that increased oxidative stress (Figure 7), likely resulting from an imbalance of intracellular $\mathrm{Ca}^{2+}$ homeostasis [25,26,28-30], is a critical early myopathic trigger in CASQ1-null mice. In support of this hypothesis, we show that treatment with NAC, a potent antioxidant, corrected the increased oxidative stress, reduced the incidence of mitochondrial damage, and improved muscle function of CASQ1-null mice (Figure 8). These results suggest that NAC (or other anti-oxidants,
$[42,47])$ could be considered as a therapeutic intervention to prevent mitochondrial damage and improve muscle function in CCD and, more generally, to mitigate the deleterious effects of increased oxidative stress and mitochondrial damage in other muscle disorders [62].

\section{Additional files}

Additional file 1: Figure S1. Age-dependent survival curve. This is a figure showing the mortality rate of male and female WT and CASQ1-null mice. Detailed description is provided within the file. 
Additional file 2: Figure S2. PGC-1a expression. This is a figure showing representative Western blot of PGC-1a of EDL total homogenates at 4 and 25 months of age. Detailed description is provided within the file.

Additional file 3: Table S1. Analysis of structural disarray. Detailed description is provided within the file.

Additional file 4: Table S2. Body and muscle weight. Detailed description is provided within the file.

Additional file 5: Table S3. Gene microarray analysis. Detailed description is provided within the file.

Additional file 6: Figure S3. Dissected EDL muscles. This is a figure showing the color of EDL muscles from WT and CASQ1-null mice. Detailed description is provided within the file.

Additional file 7: Figure S4. Mitochondrial superoxide flashes. This is a figure showing the temperature dependence of mitochondrial superoxide flash activity in the presence or absence of NAC. Detailed description is provided within the file.

\section{Abbreviations}

$\mathrm{Ca}^{2+}$ : calcium ions; CASQ1: skeletal isoform of calsequestrin; CCD: central core disease; CRU: calcium release unit; CSA: cross-sectional area; $a_{15} D H P R$ : skeletal muscle isoform of dihydropyridine receptor; DTNB: 5,5'-dithio-bis (2-nitrobenzoic acid); EC coupling: excitation-contraction coupling; EDL: extensor digitorum brevis; EM: electron microscopy; FDB: flexor digitorum brevis; GSH and GSSG: reduced and oxidized glutathione; $\mathrm{MH}$ : malignant hyperthermia; MHS: malignant hyperthermia susceptibility; mSOF: mitochondrial superoxide flashes; mt-cpYFP: mitochondria-targeted circularly permuted yellow fluorescent protein; NAC: N-acetylcysteine; O.D: optical density;

PGC-1a: peroxisome proliferator-activated receptor gamma coactivator 1-alpha; ROS: reactive oxygen species; RT: room temperature; RYR: ryanodine receptor; SOCE: store-operated $\mathrm{Ca}^{2+}$ entry; SR: sarcoplasmic reticulum;

TMRE: tetramethylrhodamine ethyl ester; TNB: 5-thio-2-nitrobenzene; T-tubule: transverse tubule; WT: wild type.

\section{Competing interests}

The authors declare that they have no competing interests.

\section{Authors' contributions}

FP, RTD, and CR initially conceived and directed the study and then supervised research progress. Each of the authors has made substantial contributions to conception/design of experiments and analysis/ interpretation of data. $C P, M Q, L W L, A M$, and $A N$ performed the experimental work and data analysis, wrote the section of Methods/Results/ Legends and assembled Figures regarding their set of experiments. In detail, CP performed EM/confocal imaging and oxidative stress experiments (GSH/ GSSG measurements), MQ performed contractile kinetics experiments, LWL performed experiments on mitochondrial superoxide flashes, AM performed long-term NAC treatment of CASQ1-null mice, and AN performed gene profile analysis and PGC-1a Western blot analysis. FP, RTD, CP, and CR assembled and revised the full manuscript, which was sent to all authors (before submission and at each step of the reviewing process) to be critically revised for important intellectual content. All authors have read and approved the final version of the manuscript.

\section{Acknowledgements}

This study was supported by a) grants from Italian Telethon ONLUS Foundation (GGP13213 to FP and CR), NIH (AR059646 to RTD and FP); b) subcontract of grant NIH-NIAMS to FP (Project R01 AR053349-06; Coordinator: S. H. Hamilton, Baylor College, TX); c) Muscular Dystrophy Association (MDA275574 to RTD and FP); and d) the Academia Dei Lincei Fund (to LWL). We also thank 1) Drs. Stefania Fulle and Rita La Rovere for the scientific and technical assistance in GSH/GSSG measurements (Figures 7A and $8 A)$, 2) Vincenzo Sorrentino for helpful scientific discussions about findings in patients carrying the N244G mutation in CASQ1, 3) Drs. Dante Tatone and Cosmo Rossi (CeSI, Center of Research on Ageing) for technical assistance with equipment and mice housing, 4) CRIBI - Biotechnology Center and Biology Department of University of Padua for the microarray analysis, and 5) Dr. Mirta Tomasi for technical assistance in the experiments of gene profile analysis and PGC-1a western blot analysis.

\section{Author details}

'CeSI - Center for Research on Ageing \& DNICS - Department of Neuroscience, Imaging and Clinical Sciences, University G. d'Annunzio, Via L. Polacchi, 11, I-66013 Chieti, Italy. ${ }^{2}$ Department of Biomedical Sciences, University of Padova, Via U. Bassi 58/B, I-35131 Padova, Italy. ${ }^{3}$ Department of Pharmacology and Physiology, University of Rochester Medical Center, 601 Elmwood Ave.,

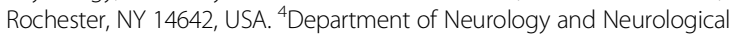
Sciences, Stanford University, 450 Serra Mall, Stanford, CA 94305, USA.

\section{Received: 28 November 2014 Accepted: 19 March 2015}

\section{Published online: 18 April 2015}

\section{References}

1. Schneider MF. Control of calcium release in functioning skeletal muscle fibers. Annu Rev Physiol. 1994:56:463-84.

2. Dulhunty AF, Beard NA, Pouliquin P, Kimura T. Novel regulators of RyR $\mathrm{Ca}^{2+}$ release channels: insight into molecular changes in genetically-linked myopathies. J Muscle Res Cell Motil. 2006;27(5-7):351-65.

3. Franzini-Armstrong C, Protasi F. Ryanodine receptors of striated muscles: a complex channel capable of multiple interactions. Physiol Rev. 1997;77(3):699-729.

4. Zhang L, Kelley J, Schmeisser G, Kobayashi YM, Jones LR. Complex formation between junctin, triadin, calsequestrin, and the ryanodine receptor: proteins of the cardiac junctional sarcoplasmic reticulum membrane. J Biol Chem. 1997;272(37):23389-97.

5. Maclennan DH, Zvaritch E. Mechanistic models for muscle diseases and disorders originating in the sarcoplasmic reticulum. Biochim Biophys Acta. 2011;1813(5):948-64. doi:10.1016/j.bbamcr.2010.11.009.

6. Galli L, Orrico A, Lorenzini S, Censini S, Falciani M, Covacci A, et al. Frequency and localization of mutations in the 106 exons of the RYR1 gene in 50 individuals with malignant hyperthermia. Hum Mutat. 2006;27(8):830. doi:10.1002/humu.9442.

7. Quane KA, Healy JM, Keating KE, Manning BM, Couch FJ, Palmucci LM, et al. Mutations in the ryanodine receptor gene in central core disease and malignant hyperthermia. Nat Genet. 1993;5(1):51-5.

8. Robinson R, Carpenter D, Shaw MA, Halsall J, Hopkins P. Mutations in RYR1 in malignant hyperthermia and central core disease. Hum Mutat. 2006;27(10):977-89.

9. Magee KR, Shy GM. A new congenital non-progressive myopathy. Brain. 1956;79(4):610-21

10. Jungbluth H. Central core disease. Orphanet J Rare Dis. 2007;2:25. doi:10.1186/1750-1172-2-25.

11. Dubowitz V, Pearse AG. Oxidative enzymes and phosphorylase in centralcore disease of muscle. Lancet. 1960;2(7140):23-4.

12. Hayashi K, Miller RG, Brownell AK. Central core disease: ultrastructure of the sarcoplasmic reticulum and T-tubules. Muscle Nerve. 1989;12(2):95-102.

13. Denborough M. Malignant hyperthermia. Lancet. 1998;352(9134):1131-6.

14. MacLennan DH, Phillips MS. Malignant hyperthermia. Science. 1992;256(5058):789-94.

15. Rosenberg H, Davis M, James D, Pollock N, Stowell K. Malignant hyperthermia. Orphanet J Rare Dis. 2007;2:21.

16. Denborough MA, Dennett $X$, Anderson RM. Central-core disease and malignant hyperpyrexia. Br Med J. 1973;1(5848):272-3.

17. Schiller HH, Mair WG. Ultrastructural changes of muscle in malignant hyperthermia. J Neurol Sci. 1974;21(1):93-100.

18. Meissner $\mathrm{G}$. Isolation and characterization of two types of sarcoplasmic reticulum vesicles. Biochim Biophys Acta. 1975;389(1):51-68.

19. MacLennan DH, Campbell KP, Reithmeier RAF. Calsequestrin. In: Calcium and cell function, vol. IV. New York: Academic Press, Inc; 1983. p. 152-73.

20. Yano K, Zarain-Herzberg A. Sarcoplasmic reticulum calsequestrins: structural and functional properties. Mol Cell Biochem. 1994;135(1):61-70.

21. Damiani $E$, Volpe $P$, Margreth A. Coexpression of two isoforms of calsequestrin in rabbit slow-twitch muscle. J Muscle Res Cell Motil. 1990;11(6):522-30.

22. Sacchetto R, Volpe P, Damiani E, Margreth A. Postnatal development of rabbit fast-twitch skeletal muscle: accumulation, isoform transition and fibre distribution of calsequestrin. J Muscle Res Cell Motil. 1993;14(6):646-53.

23. Beard NA, Sakowska MM, Dulhunty AF, Laver DR. Calsequestrin is an inhibitor of skeletal muscle ryanodine receptor calcium release channels. Biophys J. 2002:82(1 Pt 1):310-20.

24. Ikemoto $\mathrm{N}$, Ronjat M, Meszaros LG, Koshita M. Postulated role of calsequestrin in the regulation of calcium release from sarcoplasmic reticulum. Biochemistry. 1989;28(16):6764-71. 
25. Paolini C, Quarta M, Nori A, Boncompagni S, Canato M, Volpe $P$, et al. Reorganized stores and impaired calcium handling in skeletal muscle of mice lacking calsequestrin-1. J Physiol. 2007;583(Pt 2):767-84. doi:10.1113/jphysiol.2007.138024

26. Paolini C, Quarta M, D'Onofrio L, Reggiani C, Protasi F. Differential effect of calsequestrin ablation on structure and function of fast and slow skeletal muscle fibers. J Biomed Biotech. 2011;2011:634075. doi:10.1155/2011/.Epub 2011 Sep 14.

27. Royer L, Sztretye M, Manno C, Pouvreau S, Zhou J, Knollmann BC, et al. Paradoxical buffering of calcium by calsequestrin demonstrated for the calcium store of skeletal muscle. J Gen Physiol. 2010;136(3):325-38.

28. Canato M, Scorzeto M, Giacomello M, Protasi F, Reggiani C, Stienen GJ. Massive alterations of sarcoplasmic reticulum free calcium in skeletal muscle fibers lacking calsequestrin revealed by a genetically encoded probe. Proc Natl Acad Sci U S A. 2010;107(51):22326-31.

29. Dainese M, Quarta M, Lyfenko AD, Paolini C, Canato M, Reggiani C, et al. Anesthetic- and heat-induced sudden death in calsequestrin-1-knockout mice. FASEB J. 2009;23(6):1710-20

30. Protasi F, Paolini C, Canato M, Reggiani C, Quarta M. Lessons from calsequestrin-1 ablation in vivo: much more than a $\mathrm{Ca}(2+)$ buffer after all. J Muscle Res Cell Motil. 2011;32(4-5):257-70. doi:10.1007/s10974-011-9277-2.

31. Protasi F, Paolini C, Dainese M. Calsequestrin-1: a new candidate gene for malignant hyperthermia and exertional/environmental heat stroke. J Physiol. 2009;587(Pt 13):3095-100.

32. Kraeva N, Zvaritch E, Rossi AE, Goonasekera SA, Zaid H, Frodis W, et al. Novel excitation-contraction uncoupled RYR1 mutations in patients with central core disease. Neuromuscul Disord. 2013;23(2):120-32. doi:10.1016/j.nmd.2012.08.007.

33. Kraeva N, Zvaritch E, Frodis W, Sizova O, Kraev A, MacLennan DH, et al. CASQ1 gene is an unlikely candidate for malignant hyperthermia susceptibility in the North American population. Anesthesiology. 2013;118(2):344-9. doi:10.1097/ALN.0b013e318279f925.

34. Rossi D, Vezzani B, Galli L, Paolini C, Toniolo L, Pierantozzi E, et al. A mutation in the CASQ1 gene causes a vacuolar myopathy with accumulation of sarcoplasmic reticulum protein aggregates. Hum Mutat. 2014;35(10):1163-70. doi:10.1002/humu.22631.

35. Boncompagni S, Rossi AE, Micaroni M, Hamilton SL, Dirksen RT, Franzini-Armstrong $C$, et al. Characterization and temporal development of cores in a mouse model of malignant hyperthermia. Proc Natl Acad Sci U S A. 2009;106(51):21996-2001.

36. Chelu MG, Goonasekera SA, Durham WJ, Tang W, Lueck JD, Riehl J, et al. Heat- and anesthesia-induced malignant hyperthermia in an RyR1 knock-in mouse. Faseb J. 2006;20(2):329-30.

37. Gonzalez E, Messi ML, Zheng Z, Delbono O. Insulin-like growth factor-1 prevents age-related decrease in specific force and intracellular $\mathrm{Ca} 2+$ in single intact muscle fibres from transgenic mice. J Physiol. 2003;552(Pt 3):833-44. doi:10.1113/jphysiol.2003.048165.

38. Connolly AM, Keeling RM, Metha S, Pestronk A, Sanes JR. Three mouse models of muscular dystrophy: the natural history of strength and fatigue in dystrophin- dystrophin/utrophin-, and laminin alpha2-deficient mice. Neuromusc Disord. 2001;11:703-12.

39. Loud AV, Barany WC, Pack BA. Quantitative evaluation of cytoplasmic structures in electron micrographs. Lab Invest. 1965;14:996-1008.

40. Mobley BA, Eisenberg BR. Sizes of components in frog skeletal muscle measured by methods of stereology. J Gen Physiol. 1975;66(1):31-45.

41. Airey JA, Beck CF, Murakami K, Tanksley SJ, Deerinck TJ, Ellisman MH, et al. Identification and localization of two triad junctional foot protein isoforms in mature avian fast twitch skeletal muscle. J Biol Chem. 1990;265(24):14187-94.

42. Wei L, Salahura G, Boncompagni S, Kasischke KA, Protasi F, Sheu SS, et al. Mitochondrial superoxide flashes: metabolic biomarkers of skeletal muscle activity and disease. Faseb J. 2011;25(9):3068-78. doi:10.1096/fj.11-187252.

43. Rahman I, Kode A, Biswas SK. Assay for quantitative determination of glutathione and glutathione disulfide levels using enzymatic recycling method. Nat Protoc. 2006;1(6):3159-65. doi:10.1038/nprot.2006.378.

44. St-Pierre J, Drori S, Uldry M, Silvaggi JM, Rhee J, Jager S, et al. Suppression of reactive oxygen species and neurodegeneration by the PGC-1 transcriptional coactivators. Cell. 2006;127(2):397-408. doi:10.1016/j.cell.2006.09.024.

45. Lin J, Handschin C, Spiegelman BM. Metabolic control through the PGC-1 family of transcription coactivators. Cell Metab. 2005;1(6):361-70. doi:10.1016/j.cmet.2005.05.004.

46. Pellegrino MA, Desaphy JF, Brocca L, Pierno S, Camerino DC, Bottinelli R. Redox homeostasis, oxidative stress and disuse muscle atrophy. J Physio. 2011;589(Pt 9):2147-60. doi:10.1113/jphysiol.2010.203232.
47. Wang W, Fang H, Groom L, Cheng A, Zhang W, Liu J, et al. Superoxide flashes in single mitochondria. Cell. 2008;134(2):279-90. doi:10.1016/j.cell.2008.06.017.

48. Moylan JS, Reid MB. Oxidative stress, chronic disease, and muscle wasting. Muscle Nerve. 2007;35(4):411-29. doi:10.1002/mus.20743.

49. Durham WJ, Aracena-Parks P, Long C, Rossi AE, Goonasekera SA, Boncompagni S, et al. RyR1 S-nitrosylation underlies environmental heat stroke and sudden death in Y522S RyR1 knockin mice. Cell. 2008;133(1):53-65.

50. Zvaritch E, Kraeva N, Bombardier E, McCloy RA, Depreux F, Holmyard D, et al. Ca2+ dysregulation in Ryr1(14895T/wt) mice causes congenital myopathy with progressive formation of minicores, cores, and nemaline rods. Proc Natl Acad Sci U S A. 2009;106(51):21813-8. doi:10.1073/pnas.0912126106

51. Liang H, Ward WF. PGC-1alpha: a key regulator of energy metabolism. Adv Physiol Educ. 2006;30(4):145-51. doi:10.1152/advan.00052.2006.

52. Finkel T. Cell biology: a clean energy programme. Nature. 2006;444(7116):151-2. doi:10.1038/444151a.

53. Handschin C, Rhee J, Lin J, Tarr PT, Spiegelman BM. An autoregulatory loop controls peroxisome proliferator-activated receptor gamma coactivator 1alpha expression in muscle. Proc Natl Acad Sci U S A. 2003;100(12):7111-6. doi:10.1073/pnas.1232352100.

54. Suwa M, Egashira T, Nakano H, Sasaki H, Kumagai S. Metformin increases the PGC-1alpha protein and oxidative enzyme activities possibly via AMPK phosphorylation in skeletal muscle in vivo. J Appl Physiol. 2006;101(6):1685-92. doi:10.1152/japplphysiol.00255.2006.

55. Boncompagni S, Rossi AE, Micaroni M, Beznoussenko GV, Polishchuk RS, Dirksen $\mathrm{RT}$, et al. Mitochondria are linked to calcium stores in striated muscle by developmentally regulated tethering structures. Mol Biol Cell. 2009;20(3):1058-67.

56. Han D, Williams E, Cadenas E. Mitochondrial respiratory chain-dependent generation of superoxide anion and its release into the intermembrane space. Biochem J. 2001;353(Pt 2):411-6.

57. Lanner J, Georgiou DK, Dagnino-Acosta A, Ainbinder A, Cheng Q, Joshi AD, et al. AICAR prevents heat-induced sudden death in RyR1 mutant mice independent of AMPK activation. Nat Med. 2012:18(2):244-51. doi:10.1038/nm.2598.

58. Zhao X, Min CK, Ko JK, Parness J, Kimdo H, Weisleder N, et al. Increased store-operated $\mathrm{Ca}^{2+}$ entry in skeletal muscle with reduced calsequestrin-1 expression. Biophys J. 2010;99(5):1556-64.

59. Yarotskyy V, Protasi F, Dirksen RT. Accelerated activation of SOCE current in myotubes from two mouse models of anesthetic- and heat-induced sudden death. PLoS One. 2013;8(10):e77633. doi:10.1371/journal.pone.0077633.

60. Jones LR, Suzuki YJ, Wang W, Kobayashi YM, Ramesh V, Franzini-Armstrong $\mathrm{C}$, et al. Regulation of $\mathrm{Ca}^{2+}$ signaling in transgenic mouse cardiac myocytes overexpressing calsequestrin. J Clin Invest. 1998;101(7):1385-93.

61. Tomasi M, Canato M, Paolini C, Dainese M, Reggiani C, Volpe P, et al. Calsequestrin (CASQ1) rescues function and structure of calcium release units in skeletal muscles of CASQ1-null mice. Am J Physiol Cell Physiol. 2012;302(3):C575-86. doi:10.1152/ajpcell.00119.2011.

62. Dowling JJ, Arbogast S, Hur J, Nelson DD, McEvoy A, Waugh T, et al. Oxidative stress and successful antioxidant treatment in models of RYR1-related myopathy. Brain. 2012;135(Pt 4):1115-27. doi:10.1093/brain/aws036.

\section{Submit your next manuscript to BioMed Central and take full advantage of:}

- Convenient online submission

- Thorough peer review

- No space constraints or color figure charges

- Immediate publication on acceptance

- Inclusion in PubMed, CAS, Scopus and Google Scholar

- Research which is freely available for redistribution 